\title{
Dark matter and the Higgs in natural SUSY
}

\section{Aria Basirnia, Sebastian Macaluso and David Shih}

NHETC, Department of Physics and Astronomy, Rutgers University, Piscataway, NJ 08854, U.S.A.

E-mail: ariya@physics.rutgers.edu, macaluso@physics.rutgers.edu, dshih@physics.rutgers.edu

ABSTRACT: Null results from dark matter (DM) direct detection experiments and the $125 \mathrm{GeV}$ Higgs both pose serious challenges to minimal supersymmetry. In this paper, we propose a simple extension of the MSSM that economically solves both problems: a "dark sector" consisting of a singlet and a pair of SU(2) doublets. Loops of the dark sector fields help lift the Higgs mass to $125 \mathrm{GeV}$ consistent with naturalness, while the lightest fermion in the dark sector can be viable thermal relic DM, provided that it is mostly singlet. The DM relic abundance is controlled by $s$-wave annihilation to tops and Higgsinos, leading to a tight relation between the relic abundance and the spin-dependent direct detection cross section. As a result, the model will be fully probed by the next generation of direct detection experiments. Finally we discuss the discovery potential at LHC Run II.

Keywords: Supersymmetry Phenomenology

ARXIV EPRINT: 1605.08442 


\section{Contents}

1 Introduction and summary 1

2 The model $\quad 6$

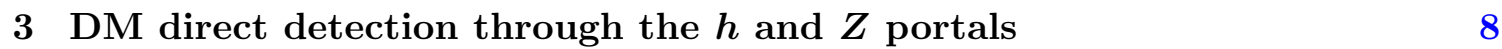

4 Higgs mass and fine-tuning $\quad 10$

5 The need for mostly-singlet DM 12

6 DM annihilation in the mostly-singlet regime $\quad 14$

$\begin{array}{lll}\text { 6.1 DM annihilation to fermions } & 15\end{array}$

$\begin{array}{ll}\text { 6.2 DM annihilation to bosons } & 16\end{array}$

$\begin{array}{ll}\text { 6.3 Total annihilation cross section } & 16\end{array}$

$\begin{array}{lll}7 & \text { Putting it all together } & 17\end{array}$

$\begin{array}{lll}7.1 & \text { Plots in the } M_{L}-M_{S} \text { plane } & 17\end{array}$

$\begin{array}{lll}\text { 7.2 Projecting onto the thermal relic contour } & 19\end{array}$

8 Outlook 21

8.1 UV considerations 21

8.2 LHC phenomenology 21

8.3 Future directions 24

A Connecting model parameters to DD cross sections $\quad 25$

B Validating SPheno one loop Higgs mass $\quad 25$

C LHC cross section analytics $\quad 26$

\section{Introduction and summary}

The MSSM paradigm is under siege from both the LHC and dark matter (DM) direct detection. The Higgs mass at tree-level in the MSSM is famously bounded by $m_{Z}$, and relying on radiative corrections from stops and other particles in the MSSM forces the stops to be either at least $\sim 10 \mathrm{TeV}$ or their $A$-terms to be multi-TeV (for recent reviews and original references, see e.g. [1-3]). Together with the null direct search results at the LHC, this puts the fine-tuning in the MSSM at the percent level or worse. Meanwhile, to evade stringent direct and indirect detection bounds, thermal relic neutralino DM in the MSSM 
must rely on increasingly contrived numerical accidents (well-tempering, blind spots, funnels, co-annihilations) or an increasingly heavy SUSY scale (e.g. $\sim 1 \mathrm{TeV}$ Higgsinos or $\sim 2-3 \mathrm{TeV}$ winos) (see e.g. [4-6] for recent comprehensive studies). The latter constitutes a DM version of the little hierarchy problem, whereby the WIMP miracle's preference for TeV-scale DM (as opposed to $100 \mathrm{GeV}$ scale DM) is in tension with naturalness.

This strongly motivates looking beyond the MSSM for both the source of the Higgs mass and dark matter. Although it is logically possible that different sectors are independently responsible for the Higgs mass and dark matter, it is interesting to contemplate more elegant and economical models where a single sector generates both. In this paper, we will study such a model. We will show how to achieve a $125 \mathrm{GeV}$ Higgs and thermal relic WIMP DM consistent with all existing constraints, while greatly ameliorating the fine-tuning, by just adding a pair of $\mathrm{SU}(2)$ doublets $L, \bar{L}$ and a singlet $S$ to the MSSM. With a $Z_{2}$ "DM parity" that keeps the lightest state in the dark sector stable, together with matter parity from the MSSM, the most general renormalizable superpotential for this "dark sector" is:

$$
W=\frac{1}{2} M_{S} S^{2}+M_{L} L \bar{L}+k_{u} H_{u} L S-k_{d} H_{d} \bar{L} S
$$

Although it would be interesting to also consider phases, we will focus on real couplings in this paper for simplicity. Then without loss of further generality, we can take $M_{S}$ and $M_{L}$ to be positive.

The idea of extending the Standard Model (SM) with a "singlet-doublet DM" sector has been studied previously in [7-14], motivated by minimality and by the fact that it is a simple generalization of the well-studied bino/Higgsino system of the MSSM. The idea of lifting the Higgs mass with loops of vector-like matter has also been well-studied [15-32]. But to our knowledge, the two ideas have never been combined before. ${ }^{1}$ Combining these two ideas leads to some important differences with previous works.

First, unlike in previous works on lifting the Higgs mass, our dark sector cannot be truly vector-like. The scalar soft mass-squareds of the dark sector must be positive in order to lift the Higgs mass, making our DM the lightest fermion in the dark sector. It cannot be a Dirac fermion, otherwise it would be ruled out by many orders of magnitude by $Z$-mediated spin-independent (SI) direct detection. Instead, we make the dark sector fermions Majorana (as shown in (1.1)) by having only one singlet and not a vector-like pair of them. This only has a minor effect on the contribution to the Higgs mass in this model, which we fully take into account. We will find that a $m_{h}=125 \mathrm{GeV}$ Higgs can be achieved with the fine-tuning coming from the DM being only $\sim 10 \%$, provided that $k_{u} \sim O(1)$.

Second, we differ from the singlet-doublet DM models in that we are supersymmetrizing everything. ${ }^{2}$ A priori, the parameter space of the entire model (MSSM+dark sector) is

\footnotetext{
${ }^{1} \mathrm{~A}$ related idea $[33,34]$ is to use vector-like matter to boost the Higgs mass while simultaneously opening up new annihilation channels for bino-like DM.

${ }^{2}$ Actually, in [8] they also added singlets and doublets to the MSSM. However, they considered soft masses purely from GMSB (whereas we are agnostic) and therefore they never have mostly-singlet fermionic DM. Moreover they fix $k_{u}=k_{d}=0.3$ whereas we have them as free parameters. Finally, they do not calculate the contribution to the Higgs mass from the dark sector.
} 
$\psi_{L}, \psi_{\bar{L}}$, superpartners

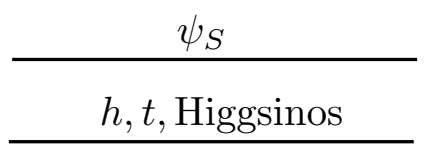

Figure 1. A typical, viable spectrum of the model. $\psi_{S}, \psi_{L}, \psi_{\bar{L}}$ are the fermionic components of the dark sector fields. Superpartners include scalar components of the dark sector and superparticles in MSSM.

vast, but most of the soft parameters do not play a significant role in the analysis. As seen in (1.1), our dark sector only couples directly to the Higgs sector and the EW gauge sector of the MSSM. We will keep the Higgsinos light $(\lesssim 300 \mathrm{GeV})$, since they contribute to the fine-tuning of the EW scale at tree level. As a result, DM annihilation to light Higgsinos through superpartners in the dark sector plays a major role in determining the relic abundance of the DM. Meanwhile, it does not change our analysis qualitatively to decouple all other MSSM superpartners (effectively at the $\sim \mathrm{TeV}$ scale). This is further motivated by the null results from the LHC. (We will remain completely agnostic as to the origin of the SUSY-breaking soft masses in this model. UV completing this with a viable messenger and hidden sector will be an interesting direction for future work.)

We will further simplify the analysis of the model by focusing on the regime where the dark matter $\chi$ is mostly-singlet, i.e. $M_{S}<M_{L}$ and $v \ll M_{L}, M_{L}-M_{S}$. As we will argue in much more detail in section 5 , this regime is absolutely necessary in order to evade direct detection bounds while raising the Higgs mass without fine-tuning. A key part of the argument, which distinguishes this from the bino/Higgsino system in the MSSM, is that $k_{u}$ must be $O(1)$ in order to lift the Higgs mass without fine-tuning. This eliminates both the well-tempered regime and the mostly-doublet regime vis a vis DM direct detection. The mostly-doublet regime is further unpromising because (by analogy with pure Higgsino DM in the MSSM) it would require a DM mass in excess of $1 \mathrm{TeV}$, and this would greatly exacerbate the fine-tuning problem, since the rest of the dark sector would have to be even heavier. This leaves the mostly-singlet regime, where the analysis of the model greatly simplifies, and we are able to understand all the features of the model with simple analytic formulas. A cartoon spectrum of the model that describes these hierarchies qualitatively is shown in figure 1 .

In this work, we will assume the simplest DM scenario, namely that $\chi$ is a thermal relic comprising all of the DM. In the mostly-singlet limit with $k_{u} \sim 1$, we will show that the thermal relic abundance is controlled by just two DM annihilation channels: $s$-wave $t \bar{t}$ (through $s$-channel $Z$ exchange) and $s$-wave Higgsinos (through $t$-channel superpartner exchange). Assuming $M_{S} \ll M_{L}$ for simplicity, we find:

$$
\sigma v_{\chi} \approx \frac{3 k_{u}^{4} m_{t}^{2}}{32 \pi M_{L}^{4}}+\frac{\left(k_{u}^{2}+k_{d}^{2}\right)^{2} \mu^{2}}{16 \pi\left(M_{L}^{2}+m^{2}\right)^{2}}
$$


where $m$ is a common soft mass for the dark sector scalars. As noted above, the second term coming from Higgsinos is a major difference from the non-supersymmetric singletdoublet DM models that have been studied previously. Having more annihilation channels increases $\sigma v_{\chi}$, making it possible to have smaller effective couplings between the DM and the SM. This opens up more parameter space that is not ruled out by direct detection experiments and yet still has the correct thermal relic abundance, as compared to the non-SUSY singlet-doublet models.

Interestingly, the DM mass drops out of the annihilation cross section (1.2) in the mostly-singlet limit. The WIMP miracle becomes one for the mediator scale, not the WIMP mass! With $k_{u} \sim k_{d} \sim 1, m \sim M_{L}$ and $\mu \lesssim 300 \mathrm{GeV}$, mediator scales of $M_{L} \sim 1-2 \mathrm{TeV}$ are implied by the thermal relic constraint. Meanwhile the DM can be much lighter than this, alleviating the DM little hierarchy problem. It is also interesting to contrast this with the mostly bino limit of the bino/Higgsino system in the MSSM. There the annihilation cross section is not large enough, being suppressed by $g_{1}^{4}$ instead of $k_{u}^{4}$. Our model (and singlet-doublet DM more generally) gets around this in the mostly-singlet regime with $O(1)$ Yukawa couplings that are free parameters, not fixed to be $g_{1}$ by supersymmetry.

Meanwhile, DM direct detection in these models is completely controlled by the effective couplings of the DM to the Higgs and $Z$ respectively:

$$
\delta \mathcal{L}=c_{h} h \bar{\psi}_{\chi} \psi_{\chi}+c_{Z} Z_{\mu} \bar{\psi}_{\chi} \gamma^{\mu} \gamma^{5} \psi_{\chi}
$$

As is well-known, $c_{h}\left(c_{Z}\right)$ controls the SI (SD) direct detection cross section. For direct detection, as we will review, the current best bounds for our DM mass range of interest $\left(100 \lesssim m_{\mathrm{DM}} \lesssim 1000 \mathrm{GeV}\right)$ come from LUX [35] and IceCube [36]. We will convert the official experimental results, which are phrased in terms of the DM-nucleon cross section, into limits on $c_{h}$ and $c_{Z}$. Furthermore, in the mostly-singlet limit, we will obtain simple analytic expressions for $c_{h}$ and $c_{Z}$. We will see that $c_{h}$ can be naturally small enough for mostly-singlet DM, due to suppression from the heavier doublet scale, as well as a mild blind-spot cancellation:

$$
c_{h} \approx-\frac{m_{\chi}+\frac{2 k_{d} M_{L}}{k_{u} \tan \beta}}{\sqrt{2} v} \frac{k_{u}^{2} v^{2}}{M_{L}^{2}}
$$

provided that $k_{u} \sim k_{d}$. We should emphasize here that the Higgs mass depends not just on $c_{h}$ but also on the effective Yukawa couplings between the Higgs and the other dark sector particles. So even dialing $c_{h} \rightarrow 0$ does not qualitatively affect the Higgs mass calculation. Meanwhile $c_{Z}$ is given in the mostly-singlet limit by:

$$
c_{Z} \approx-\frac{g_{2}}{4 c_{W}} \frac{k_{u}^{2} v^{2}}{M_{L}^{2}}
$$

According to our discussion above, after fixing the thermal relic density constraint $\Omega_{\mathrm{DM}}^{\mathrm{obs}} h^{2} \approx$ $0.12, c_{Z}$ is essentially fixed to lie within a narrow range which depends primarily on the Higgsino mass $\mu$. Therefore imposing the relic density constraint essentially fixes the SD cross section. Fortunately, this value is not ruled out yet, but the next generation of DM experiments (e.g. Xenon1T [37], LZ [38]) should completely rule out or discover this model. 
Although direct detection is controlled by $c_{Z}$ and $c_{h}$, the other facets of the model (relic abundance, Higgs mass) depend on more than just these couplings, so our model does not fit completely into the framework of $Z$ - and $h$-portal DM. For instance, we mentioned above that the Higgsino cross section arises entirely from $t$-channel superpartner exchange. Also, we find that DM annihilation to dibosons is suppressed more than would be the case in $Z$ and $h$ portal models, in part due to $t$-channel exchange of doublet fermions. Similar comments apply to the effective operator formalism: our DM is generally not light enough compared to the mediator scale (the doublet mass) for the annihilation to be accurately captured by effective operators. Evidently, the complete model (1.1) is required for an accurate analysis. This illustrates the shortcomings and limitations of both simplified models and effective operator approaches to dark matter.

We have focused primarily on the standard direct detection searches in this work, because other indirect probes of our dark sector are far less sensitive. For example, the Fermi experiment and others have searched for energetic photons produced through DM annihilating at the centers of dwarf galaxies. For DM masses above $100 \mathrm{GeV}$, Fermi does not constrain any point with the right relic-abundance [39], assuming (as is the case for us) that the relic abundance is determined by $s$-wave annihilation. Meanwhile, searches at colliders and electroweak precision tests (EWPT) could have put constraints on our model. However as we will discuss further in section 8.2, LHC bounds [40-43] on $c_{h}$ and $c_{Z}$ from monojets+MET and monophoton+MET are orders of magnitude weaker than direct detection for the range of DM masses that we are interested in. We will briefly discuss mono- $(W, Z, h)+\mathrm{MET}$ and show how it could probe the low end of DM masses $\left(m_{\mathrm{DM}} \sim 200 \mathrm{GeV}\right)$ in our model, with $300 / \mathrm{fb}$ at LHC Run II. Finally, limits from Higgs and $Z$ invisible width do not apply to the mass range of DM that we consider in this work, and we checked that contributions to the $S$ and $T$ parameters are well within the acceptable range, in agreement with previous studies of these variables in closely-related models $[11,19]$.

In this paper, we will analyze the model using a combination of simple, approximate analytic expressions valid in the mostly-singlet regime, and more precise numerical methods that take into account the full suite of one (and even two) loop threshold corrections. The analytic approach, while being reasonably accurate, is primarily a source of intuition and understanding. The numerical approach is meant to be more accurate and to provide us with the quantitative results. Clearly, having both numerics and analytics is a vital source of cross-checks, giving us greater confidence in our results.

Our numerical methods are based on publicly available codes. Our starting point was the powerful SARAH 4.5.8 framework [44] for automated analysis of general models. Once we properly defined our model, SARAH automatically generated source code for SPheno 3.3.7 [45, 46] and for micrOMEGAs 4.1.8 [47]. ${ }^{3}$ The former calculates the spectrum while the latter calculates the DM relic abundance and direct detection cross sections. In our numerical calculations, all MSSM soft masses as well as gauginos are taken to be at $1 \mathrm{TeV}$,

\footnotetext{
${ }^{3}$ We are extremely grateful to Florian Staub for his time and patience in helping us set up the SARAH model and link it to these other codes.
} 
and the $A$-terms are set to zero. As noted earlier, since $\mu$ appears at tree level in finetuning of the electroweak scale we treat it differently. We pick $\mu=300 \mathrm{GeV}$ in our numerical calculations which corresponds roughly to $10 \%$ fine-tuning. We also consider $\mu=100 \mathrm{GeV}$ to see the effect of $\mu$ on our analysis. Finally, to saturate the tree level contribution to the Higgs mass, we take the other Higgses to be heavy and in the decoupling limit, and we take $\tan \beta=10$.

The outline of our paper is as follows. In section 2 we introduce the model. Then in section 3, we derive direct detection limits from LUX and IceCube on the effective couplings $c_{h}$ and $c_{Z}$. We will emphasize that these results are general and are not limited to the model we consider in this work. In section 4 we compute the one-loop corrections to the Higgs mass from the new particles in our model, and we discuss fine-tuning. We argue in section 5 that the mostly singlet case is the only viable scenario. In the mostly singlet limit, we provide analytic expressions for dark matter annihilation in the early universe for our model in section 6 . In section 7 we put everything together to show the viable parameter space that satisfies all direct detection constraints while having the right relic abundance and Higgs mass. Here we demonstrate quantitatively that requiring $\chi$ to be all of the DM essentially fixes $c_{Z}$ (and hence $\sigma^{\mathrm{SD}}$ ) to a unique value which is not yet ruled out by direct detection, but will be fully within reach of the next generation of experiments. We conclude by studying the collider signatures for LHC Run II and the UV behavior of the model, and giving suggestions on future directions on section 8. Technical details and validations are reserved for three appendices. In appendix A we review the derivation of the direct detection cross sections from effective DM nucleon couplings. We validate our numerical and analytical calculations of the Higgs mass in appendix B. Finally we provide analytical cross sections for DM production at LHC II in appendix C.

\section{The model}

We begin by describing the model in more detail. We add to the MSSM a "dark sector" consisting of a vector-like pair of $\mathrm{SU}(2)$ doublets $L, \bar{L}$ and a gauge singlet $S{ }^{4}$ The dark sector is equipped with an unbroken $Z_{2}^{\mathrm{DM}}$ parity symmetry under which all new fields are odd and all MSSM fields are even. This makes the lightest new state stable and a DM candidate. Finally, we assume MSSM matter parity, under which all the dark sector fields have the same charge; otherwise there will be additional, potentially dangerous terms. ${ }^{5}$ The transformation properties of the dark sector under the gauge and global symmetries is summarized in table 1.

The most generic superpotential consistent with these symmetries is:

$$
\delta W=\frac{1}{2} M_{S} S^{2}+M_{L} L \bar{L}+k_{u} H_{u} L S-k_{d} H_{d} \bar{L} S
$$

\footnotetext{
${ }^{4}$ To keep gauge coupling unification as in MSSM, we can assume $L$ and $\bar{L}$ are part of complete $\mathbf{5}$ and $\overline{\mathbf{5}}$ multiplets of SU(5). We take their colored partners to be heavy and decoupled for simplicity.

${ }^{5}$ The assumption of matter parity implies another stable particle - either the LSP in the MSSM, or the gravitino. Either way, we assume the parameters and cosmological history are such that this will add a negligible additional component to the dark matter relic density.
} 


\begin{tabular}{|c|c|c|c|c|c|}
\hline & $\mathrm{SU}(3)_{c}$ & $\mathrm{SU}(2)_{L}$ & $\mathrm{U}(1)_{Y}$ & $Z_{2}^{\mathrm{DM}}$ & $Z_{2}^{M}$ \\
\hline$L$ & $\mathbf{1}$ & $\mathbf{2}$ & $-\frac{1}{2}$ & -1 & 1 \\
$\bar{L}$ & $\mathbf{1}$ & $\mathbf{2}$ & $\frac{1}{2}$ & -1 & 1 \\
$S$ & $\mathbf{1}$ & $\mathbf{1}$ & 0 & -1 & 1 \\
\hline
\end{tabular}

Table 1. Gauge and global symmetries of the dark sector.

The superpotential has four new parameters in addition to the MSSM: $M_{L}, M_{S}, k_{u}, k_{d}$. There is one physical complex phase, but as discussed in the introduction, we will take these parameters to be real in this paper. In this case, there is still a physical sign. We will take $M_{L}, M_{S}$ and $k_{u}$ to be positive and put the sign into $k_{d}$.

For the soft SUSY-breaking Lagrangian, for simplicity we take the minimal case with equal soft mass-squareds and no $A$ - or $B$-terms:

$$
\delta \mathcal{L}_{\text {soft }}=-m^{2}\left(|\bar{\ell}|^{2}+|\ell|^{2}+|s|^{2}\right)
$$

(We denote the scalar components of the dark sector superfields with lowercase letters.) Allowing different soft masses for the different fields will not change most of the discussion in this paper, only the contributions to Higgs mass.

As we want this new sector to increase the lightest Higgs mass analogous to the MSSM stops, we assume that $m^{2}>0$. This implies that the DM candidate is a fermion. Furthermore it is Majorana, thanks to the fact that we have included only one singlet in the theory. Had we started with a Dirac pair of $S$ and $\bar{S}$ and defined the mass term as $M_{S} S \bar{S}$, our dark matter would have had a vector-like coupling to the $Z$. In that case it would have been impossible to hide it from SI direct detection experiments while keeping the interesting features of our model.

After EWSB, neutral fields in the dark sector mix through the Yukawa couplings in (2.1). The fermion mass matrix of the neutral states is:

$$
\mathcal{M}=\left(\begin{array}{ccc}
M_{S} & \hat{k}_{u} v & \hat{k}_{d} v \\
\hat{k}_{u} v & 0 & M_{L} \\
\hat{k}_{d} v & M_{L} & 0
\end{array}\right)
$$

where we have introduced $\hat{k}_{u} \equiv k_{u} \sin \beta$ and $\hat{k}_{d} \equiv k_{d} \cos \beta$, with $\tan \beta=v_{u} / v_{d}$ and $v_{u}^{2}+v_{d}^{2}=$ $v^{2} \approx(174 \mathrm{GeV})^{2}$ as usual. We take large $\tan \beta=10$ in this paper to saturate the upper bound on the tree level Higgs mass. The mass matrix is diagonalized by $U \mathcal{M} U^{\dagger}=\mathcal{M}_{\text {diag }}$. The spectrum of the model consists of three Majorana fermions with masses $m_{\chi_{1}}<m_{\chi_{2}}<$ $m_{\chi_{3}}$ and a Dirac charged fermion with mass $m_{\chi^{ \pm}}=M_{L}$. The dark matter candidate is then $\chi \equiv \chi_{1}$.

We note that the fermionic part of our dark sector is analogous to Bino-Higgsino DM in the MSSM (with everything else decoupled), except that in the Bino-Higgsino system, we effectively have $k_{u}=k_{d}=g^{\prime} / \sqrt{2}$, whereas here $k_{u}$ and $k_{d}$ are general. In fact, as discussed in the introduction, here we will be primarily interested in $k_{u}, k_{d} \sim O(1)$. 
After rotating to the mass eigenbasis, DM- $Z$ and DM-Higgs couplings are generated:

$$
\delta \mathcal{L}=c_{h} h \bar{\psi}_{\chi} \psi_{\chi}+c_{Z} Z_{\mu} \bar{\psi}_{\chi} \gamma^{\mu} \gamma^{5} \psi_{\chi}
$$

where $\psi_{\chi}=\left(\chi, \chi^{\dagger}\right)^{T}$ is a 4-component Majorana fermion and $c_{h}$ and $c_{Z}$ are given by:

$$
\begin{aligned}
c_{h} & =\frac{1}{\sqrt{2}} \operatorname{Re}\left(\hat{k}_{u} U_{11}^{*} U_{12}^{*}+\hat{k}_{d} U_{11}^{*} U_{13}^{*}\right) \\
& =\frac{v}{\sqrt{2}}\left(\frac{m_{\chi}\left(\hat{k}_{d}^{2}+\hat{k}_{u}^{2}\right)+2 \hat{k}_{d} \hat{k}_{u} M_{L}}{M_{L}^{2}+2 M_{S} m_{\chi}-3 m_{\chi}^{2}+v^{2}\left(\hat{k}_{d}^{2}+\hat{k}_{u}^{2}\right)}\right)
\end{aligned}
$$

and

$$
\begin{aligned}
c_{Z} & =\frac{g_{2}}{4 c_{W}}\left(\left|U_{12}\right|^{2}-\left|U_{13}\right|^{2}\right) \\
& =\frac{g_{2}}{4 c_{W}} \frac{\left(M_{L}^{2}-m_{\chi}^{2}\right) v^{2}\left(\hat{k}_{d}^{2}-\hat{k}_{u}^{2}\right)}{\left(M_{L}^{2}-m_{\chi}^{2}\right)^{2}+v^{2}\left(\left(\hat{k}_{u}^{2}+\hat{k}_{d}^{2}\right)\left(M_{L}^{2}+m_{\chi}^{2}\right)+4 \hat{k}_{d} \hat{k}_{u} M_{L} m_{\chi}\right)}
\end{aligned}
$$

As is well-known [9-13], $c_{h}$ and $c_{Z}$ play an important role in the analysis of singlet-doublet DM: they are entirely responsible for SI and SD direct detection, respectively. In the next section, we will review the current direct detection constraints on $c_{h}$ and $c_{Z}$.

\section{$3 \quad$ DM direct detection through the $h$ and $Z$ portals}

In the DM mass range of interest $\left(100 \mathrm{GeV} \lesssim m_{\mathrm{DM}} \lesssim 1 \mathrm{TeV}\right)$, the LUX experiment currently sets the best bound on SI elastic WIMP-nucleon scattering [35]. Meanwhile, the best limits for SD elastic WIMP-proton (WIMP-neutron) scattering come from IceCube [36] and LUX [35]. The IceCube limits depend on an assumption of DM $s$-wave annihilation in the sun exclusively to a single SM final state. As we will show in section 6, our DM annihilates in the $s$-wave to both $t \bar{t}$ and Higgsinos. Annihilation to Higgsinos could weaken the limits somewhat if the Higgsinos are stable, but that depends in detail on the other parameters of the model (such as $\mu, k_{u}$ and $k_{d}$ ). Here we consider the simplest case where annihilation is only to $t \bar{t}$; this will provide the "worst case scenario" where the SD bound from IceCube is strongest. In section 7 we will also take into account annihilation to stable Higgsinos.

In this section, we will recast these constraints in terms of the couplings $c_{h}$ and $c_{Z}$. The discussion here can be viewed as an update of the nice treatment in [4] with the latest experimental results (in particular LUX). It is worth emphasizing that these bounds on $c_{h}$ and $c_{Z}$ are quite model independent. Any WIMP DM that couples to SM mainly through Higgs and $Z$ (including MSSM neutralinos) should satisfy these bounds.

To convert the results of these experiments into bounds on $c_{Z}$ and $c_{h}$, we first translate $c_{Z}$ and $c_{h}$ into the couplings appearing in the effective Lagrangian for direct detection:

$$
\delta \mathcal{L} \supset \sum_{q}\left(\xi_{q}^{\mathrm{SI}}\left(\bar{\psi}_{\chi} \psi_{\chi}\right)(\bar{q} q)+\xi_{q}^{\mathrm{SD}}\left(\bar{\psi}_{\chi} \gamma^{\mu} \gamma_{5} \psi_{\chi}\right)\left(\bar{q} \gamma_{\mu} \gamma_{5} q\right)\right)
$$



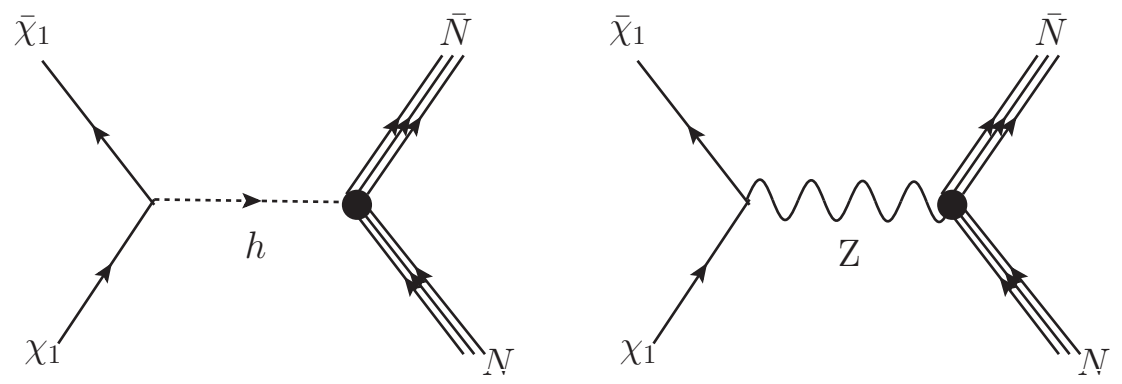

Figure 2. Higgs $(Z)$ exchange diagrams contributing to SI (SD) cross sections.

In Higgs and $Z$-portal DM models, the SI (SD) terms arise from Higgs $(Z)$ exchange, as shown in figure 2. The coefficients of the effective operators are given in terms of $c_{h}$ and $c_{Z}$ as:

$$
\xi_{q}^{\mathrm{SI}}=y_{q} \frac{c_{h}}{m_{h}^{2}}, \quad \xi_{q}^{\mathrm{SD}}=\frac{g_{2} \eta_{q}}{4 c_{W}} \frac{c_{Z}}{m_{Z}^{2}}
$$

with $y_{q}$ being the Yukawa coupling and $\eta_{q}=1(-1)$ for down-type (up-type) quarks.

Then we use standard formulas that relate the DM-nucleon cross sections to $\xi_{q}^{\mathrm{SI}, \mathrm{SD}}$ (see appendix A for our conventions and parameter choices). The result, assuming $m_{\mathrm{DM}} \gg m_{p, n}$ is given by:

$$
\begin{aligned}
\sigma^{\mathrm{SI}} & =c_{h}^{2} \times\left(2.11 \times 10^{3} \mathrm{zb}\right) \\
\sigma_{p}^{\mathrm{SD}} & =c_{Z}^{2} \times\left(1.17 \times 10^{9} \mathrm{zb}\right) \\
\sigma_{n}^{\mathrm{SD}} & =c_{Z}^{2} \times\left(8.97 \times 10^{8} \mathrm{zb}\right)
\end{aligned}
$$

In principle, $\sigma_{p}^{\mathrm{SI}}$ and $\sigma_{n}^{\mathrm{SI}}$ are slightly different but the difference is negligible, so we only take $\sigma_{p}^{\mathrm{SI}}$ to represent both.

The resulting limits on $c_{h}$ and $c_{Z}$ are shown in figure $3 .{ }^{6}$ Amusingly, we note that although the constraint on the SI cross section is $\sim 10^{5}$ stronger than the SD cross-section, translated constraints on $c_{h}$ and $c_{Z}$ are of the same order of magnitude. This is because the Higgs-nucleon effective Yukawa coupling $\left(y_{h N N}\right)$ is much weaker than the $Z$-nucleon effective coupling $\left(\sim g_{2}\right)$. Recall that the Higgs-nucleon coupling is mainly due to Higgsgluon-gluon loop-induced interaction with heavy quarks running in the loop

$$
y_{h N N}=\frac{\sqrt{2} \alpha_{s} N_{H}}{24 \pi v}\left\langle N\left|G^{a \mu \nu} G_{\mu \nu}^{a}\right| N\right\rangle=\frac{\sqrt{2} N_{H} m_{N}}{3 b v} \simeq 10^{-3}
$$

where $N_{H}=3$ is the number of heavy quarks and $b=11-\frac{2}{3} N_{H}$ comes from QCD beta function at one loop. The second equality can be calculated using QCD scale anomaly that relates the QCD beta function to nucleon mass (see [50] for the original references).

\footnotetext{
${ }^{6}$ We agree with the limits from [4] after taking into account a factor of 2 in both $c_{Z}$ and $c_{h}$ from 4 component vs. 2-component notation. We also agree with limits on operators from [48] modulo a factor of 4 between Dirac and Majorana fermions and a factor of a few difference between [48] and the latest LUX bounds. We do not agree with the limits on $g_{A}$ (related to our $c_{Z}$ via $g_{A}=\frac{c_{w}}{g_{2}} c_{Z}$ ) reported in figure 3 of [49]. Their limit on $g_{A}$, derived from essentially the same LUX results, is over an order of magnitude weaker than ours.
} 


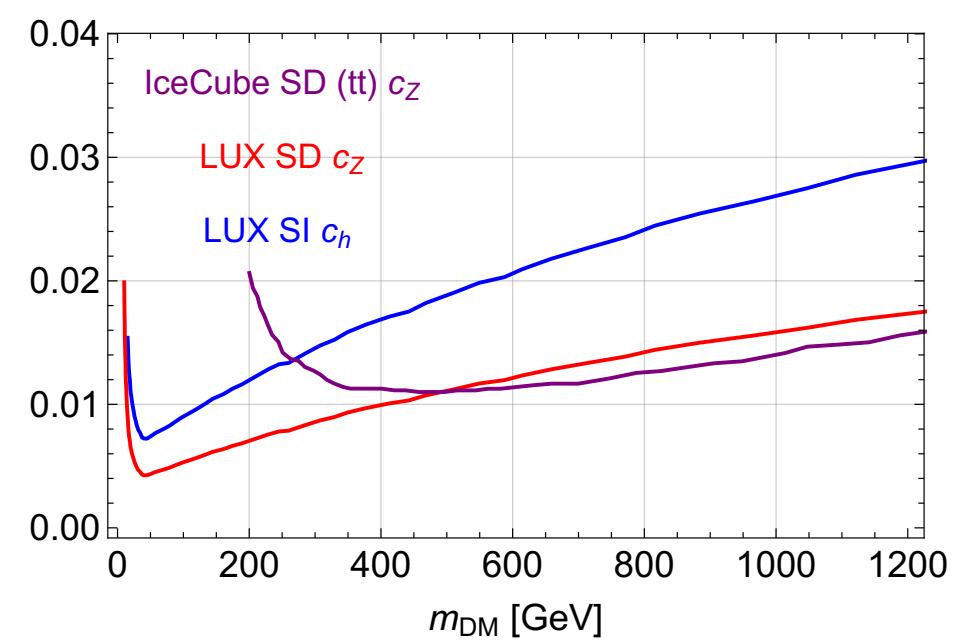

Figure 3. Limits on $c_{h}$ from LUX [35] (blue) and $c_{Z}$ from LUX [35] (red) and IceCube $t \bar{t}$ [36] supposing DM only annihilates to $t \bar{t}$ (purple).

\section{Higgs mass and fine-tuning}

In this section we will describe our calculation of the Higgs mass in the model and its implication for the fine-tuning of the EW scale. As described in the introduction, we used SARAH 4.5.8 [44] and SPheno 3.3.7 [45, 46] to include all the loop corrections (contributions up to two loops both from the MSSM and the dark sector [51, 52]). Here we will describe an analytic treatment of the dominant one-loop contributions from $k_{u}$ and $k_{d}$. This will serve as a valuable source of intuition, as well as a validation of the full two-loop numerical calculation (for more details on the validation, see appendix B).

The one-loop Higgs mass was previously computed in the literature using the ColemanWeinberg potential in closely-related vector-like extensions of the MSSM [17-19, 21]. However, there are some key differences with our case that necessitate a fresh look. First, as noted above, in these past works, the vector-like extension was Dirac, while ours is Majorana (the difference between $W \supset M S \tilde{S}$ and $W \supset \frac{1}{2} M S^{2}$ ). This leads to small differences in the formula for the Higgs mass. Second, previous works presented analytic formulas for the one-loop Higgs mass only in the simplified limit with common fermion masses $\left(M_{L}=M_{S}\right)$. Motivated by the DM side of the story, we will need the Higgs mass in a rather different regime, the mostly-singlet regime where $M_{S} \ll M_{L}$.

Other effects that we will ignore in our discussion here, but that are taken into account in the full numerical SARAH-SPheno calculation, include $g_{1,2}^{2}$ corrections, two-loop corrections, and the effective $A$-terms due to $\mu$. The effects of $g^{2}$ are about a $10-20 \%$ correction to $\delta m_{h}^{2}$, which amounts to a $2 \mathrm{GeV}$ shift in $m_{h}$. That matters for our calculations quantitively but not qualitatively. The $\mu$ values we consider in this paper motivated by naturalness are small enough that $\mu$ has a negligible effect on the Higgs mass. Finally, we are interested in moderately-large values of $\tan \beta$ (e.g. $\tan \beta=10$ ) but for simplicity we will present the $\tan \beta \rightarrow \infty$ limit here. The corrections due to $1 / \tan \beta$ also do not make a qualitative difference. (In particular, there are no blind-spot cancellations here.) 
With all of these simplifying assumptions, the result of our one-loop Coleman-Weinberg calculation is:

$$
\delta m_{h}^{2}=\frac{1}{4 \pi^{2}} k_{u}^{4} v^{2}\left(f_{1} \log \left(1+x_{L}^{2}\right)+f_{2} \log \left(1+x_{S}^{2}\right)+f_{3} \log \frac{x_{S}^{2}}{x_{L}^{2}}\right)
$$

with

$$
\begin{aligned}
f_{1} & =\frac{\left(2 x_{L}^{4}+x_{L}^{6}+3 x_{L}^{2} x_{S}^{2}+3 x_{L}^{4} x_{S}^{2}-x_{S}^{4}\right) x_{S}^{2}}{\left(x_{L}^{2}-x_{S}^{2}\right)^{3}} \\
f_{2} & =\frac{\left(x_{L}^{2}-5 x_{S}^{2}-x_{L}^{2} x_{S}^{2}-3 x_{S}^{4}\right) x_{L}^{4}}{\left(x_{L}^{2}-x_{S}^{2}\right)^{3}} \\
f_{3} & =\frac{x_{L}^{4} x_{S}^{2}\left(x_{L}^{2}+3 x_{S}^{2}\right)}{\left(x_{L}^{2}-x_{S}^{2}\right)^{3}}
\end{aligned}
$$

where $x_{L}=m / M_{L}$ and $x_{S}=m / M_{S}$. A plot of $k_{u}^{-4} \delta m_{h}^{2}$ is shown in figure 4 (left). We see that $\delta m_{h}^{2}$ asymptotes to a finite value as $x_{L} \rightarrow \infty$ or $x_{S} \rightarrow \infty$. In these limits (corresponding to mostly-doublet and mostly-singlet DM respectively), the dependence on the DM mass drops out, and $\delta m_{h}^{2}$ is controlled by the ratio of the soft mass to the heavier mediator scale $\left(M_{S}\right.$ or $M_{L}$ respectively).

To raise the Higgs to $125 \mathrm{GeV}$ in this paper, we rely on a combination of the extra vector-like matter and MSSM stops. For stops at $1 \mathrm{TeV}$, which satisfy the current experimental bounds and imply about a $\sim 10 \%$ tuning of the EW VEV, the MSSM contribution to the Higgs mass is about $110 \mathrm{GeV}$ (for a recent review see e.g. [3]). Therefore the target for $\delta m_{h}^{2}$ from the dark sector is:

$$
\delta m_{h}^{2} \approx 3500 \mathrm{GeV}^{2}
$$

This selects out a contour in the $\left(x_{S}, x_{L}\right)$ plane as shown in figure 4 (left), according to the value of $k_{u}$.

This has the following implications for the fine-tuning of the EW scale. Just as the dark sector lifts the physical Higgs mass analogous to stops in the MSSM, it also contributes to the fine-tuning of the EW scale through the renormalization of $m_{H_{u}}^{2}$. Following [53, 54], we define the measure of fine-tuning to be:

$$
\Delta=\frac{2 \delta m_{H_{u}}^{2}}{m_{h}^{2}}
$$

where $\delta m_{H_{u}}^{2}$ is the running of $m_{H_{u}}^{2}$ due to the new fields

$$
\delta m_{H_{u}}^{2}=\frac{k_{u}^{2} m^{2}}{8 \pi^{2}} \log \frac{\Lambda_{\mathrm{UV}}}{\Lambda_{\mathrm{IR}}}
$$

Optimistically we take $\Lambda_{\mathrm{UV}}=10 \Lambda_{\mathrm{IR}} \sim 10 \mathrm{TeV}$. We can combine this with (4.1) and (4.3) as follows. For a given value of $k_{u}$ and a given point in the $\left(M_{S}, M_{L}\right)$ plane, we can solve (4.3) for the soft mass $m$. Then substituting this into (4.4), we get a value for $\Delta$. Regions of $\Delta \leq 20$ are shown in figure 4 (right) for different representative values of $k_{u}$. We see that we need $k_{u} \gtrsim 1$ to have any viable parameter space at all for a natural SUSY 

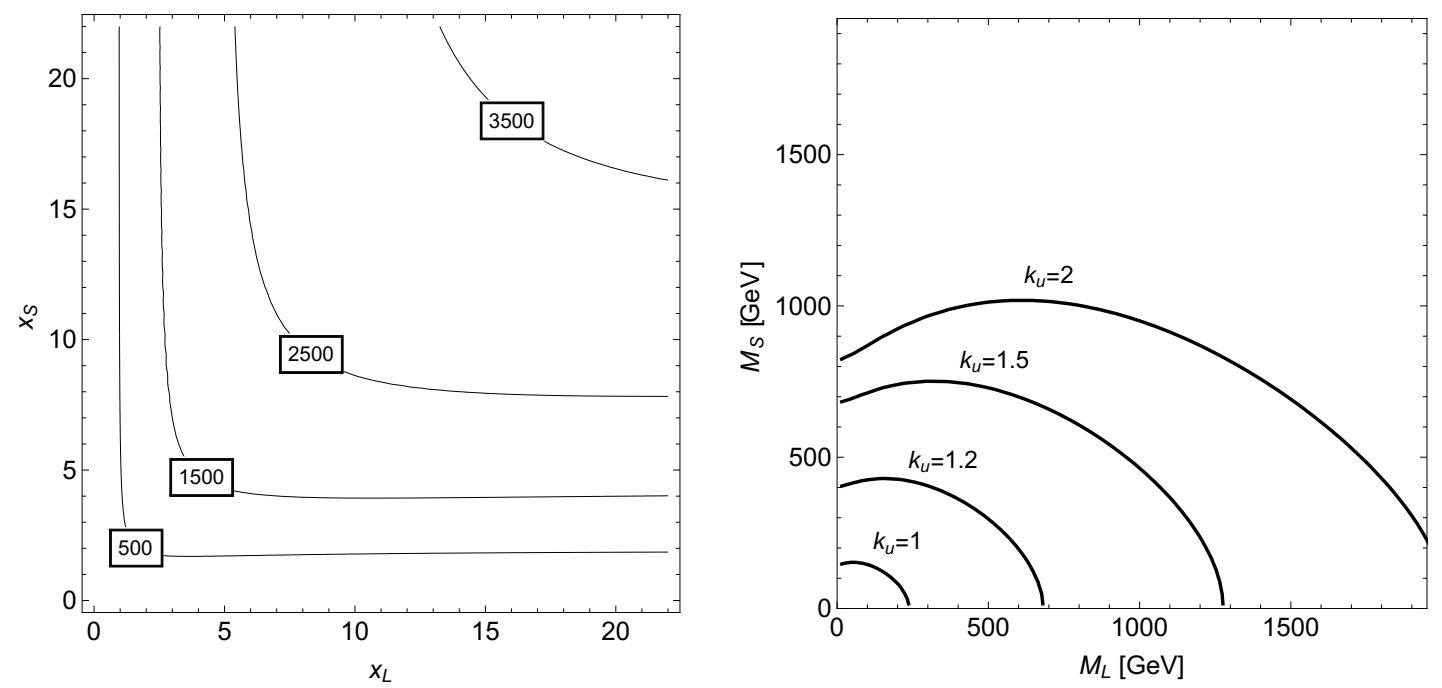

Figure 4. Left: contours of $k_{u}^{-4} \delta m_{h}^{2}$ in $\mathrm{GeV}^{2}$ in the $\left(x_{L}, x_{S}\right)$ plane, according to (4.1). Right: contours of $\Delta=20$ in the $\left(M_{L}, M_{S}\right)$ plane for different values of $k_{u}$ that have a Higgs mass at $125 \mathrm{GeV}$.

$125 \mathrm{GeV}$ Higgs. This is not surprising, since from (4.1), we see that $k_{u}$ plays the role that $y_{t}$ plays for the MSSM stops. Of course, corrections we have neglected such as the $D$-terms and two-loop effects will modify this quantitatively. However, we will see that the same qualitative implications for fine-tuning and $k_{u}$ will persist in our final plots.

\section{The need for mostly-singlet DM}

In section 2, we derived formulas for $c_{h}$ and $c_{Z}$ in terms of the parameters of the model, while in section 3 we showed that direct detection limits on $c_{h}$ and $c_{Z}$ are at the $O\left(10^{-2}\right)$ level. Finally, in section 4 , we argued that we need $k_{u} \gtrsim 1$ in order to have any viable parameter space for a natural SUSY Higgs at $125 \mathrm{GeV}$. Here we will combine these facts and show that the DM must be mostly singlet in order to be consistent with all the constraints.

Basically there are three possibilities: the well-tempered regime where $\left|M_{L}-M_{S}\right| \lesssim v$ (recall our convention is that $M_{S}$ and $M_{L}$ are positive), the mostly-doublet regime where $M_{L}<M_{S}$ and $v \ll M_{S}, M_{S}-M_{L}$, and the mostly-singlet regime where $M_{S}<M_{L}$ and $v \ll M_{L}, M_{L}-M_{S}$. Keeping in mind that we need $k_{u} \gtrsim 1$ and large $\tan \beta$ for a natural Higgs mass, the challenge is to decrease $c_{h}$ and $c_{Z}$ to the $10^{-2}$ level. In fact, $c_{h}$ alone is enough to rule out all but the mostly-singlet case. We will comment on the implications for $c_{Z}$ in sections 6 and 7 .

Examining the formula for $c_{h}(2.5)$, we see that for $\left|M_{L}-M_{S}\right| \sim v$ and $\hat{k}_{u} \sim 1$, we have $c_{h} \sim O(1)$. (In particular, there is a cancellation in the denominator, leaving it $O\left(v M_{S}\right)$ ). This rules out the well-tempered case.

The mostly-doublet case is ruled out separately by two independent considerations. First, from figure 4, we see that in order to be natural and mostly-doublet, we must have the DM mass below $\sim 800 \mathrm{GeV}$. However, we know by analogy with pure Higgsinos in the 
MSSM that the thermal relic density constraint requires $M_{L} \geq 1 \mathrm{TeV}$. (The mostly-doublet DM in this model has additional annihilation modes due to $k_{u}$ and $k_{d}$, so $M_{L}$ will be even larger.) So the mostly-doublet scenario is not promising for naturalness.

Also, from direct detection, we are basically forced into the mostly-singlet regime. In order to lower $c_{h}$ by two orders of magnitude, we must either (a) raise $M_{L}$ or $M_{S}$ to increase the denominator of (2.5), or (b) cancel the two terms in the numerator of (2.5).

(a) Increasing the denominator of (2.5) necessitates either $M_{L}$ or $M_{S} \gg v$. In the former, corresponding to mostly-singlet DM, we see that $c_{h} \propto 1 / M_{L}^{2}$ and we can achieve the required level of suppression for $M_{L} \sim 1-2 \mathrm{TeV}$ for $M_{S} \sim v$ and $k_{u} \sim 1$. Meanwhile for the latter, corresponding to mostly-doublet DM, we see that $c_{h} \propto 1 / M_{S}$ and therefore much larger $M_{S} \sim 2-5 \mathrm{TeV}$ is required for $M_{L} \sim v$ and $k_{u} \sim 1$. The latter is greatly disfavored by naturalness (it would likely be as fine-tuned as $10 \mathrm{TeV}$ stops in the MSSM).

(b) Cancelling the two terms in the numerator requires

$$
\frac{M_{L}}{m_{\chi}} \sim-\frac{1}{2} \frac{k_{u}}{k_{d}} \tan \beta
$$

This is the blind spot. Since $k_{u} \gtrsim 1$ and we are in the large $\tan \beta$ limit, the r.h.s. is generally much greater than one for any reasonable value of $k_{d}$. Therefore we must be in the mostly-singlet DM regime to realize the blind spot.

We conclude that several different constraints independently point at mostly-singlet DM as the only viable possibility.

For later reference we exhibit $c_{h}$ and $c_{Z}$ in the mostly-singlet limit

$$
\begin{aligned}
c_{h} & =-\frac{m_{\chi}+\frac{2 k_{d} M_{L}}{k_{u} \tan \beta}}{\sqrt{2} v} \frac{k_{u}^{2} v^{2}}{M_{L}^{2}}+\ldots \\
c_{Z} & =-\frac{g_{2}}{4 c_{W}} \frac{k_{u}^{2} v^{2}}{M_{L}^{2}}+\ldots
\end{aligned}
$$

Here we have taken $M_{L} \rightarrow \infty$ and $\tan \beta \rightarrow \infty$ holding fixed $M_{L} / \tan \beta$ and all the other mass scales. In figure 5 , we exhibit the amount of blind spot cancellation that is required by the SI bounds, for a typical choice of parameters that will lead to a viable relic density. We show this behavior by varying $k_{d}$ keeping other parameters fixed. We can see that the constraint on $c_{h}$ is satisfied in a fairly wide window of $k_{d}$ values $\left(-1.8 \leq k_{d} \leq-1\right)$. As for the two terms in $c_{h}$ in (5.2), this amounts to a mild cancellation at the level of one part in $3-4$. This could be viewed as another source of fine-tuning in the model, but it will be in general subdominant to the tuning of the EW scale, so we will not comment on it further. Most of the suppression of $c_{h}$ is coming from large $M_{L}$, which as we will see in the next section is fixed by the thermal relic abundance constraint.

In the same mostly-singlet regime, we also exhibit $\delta m_{h}^{2}$ :

$$
\delta m_{h}^{2}=\frac{k_{u}^{4} v^{2}}{4 \pi^{2}} \log \left(1+x_{L}^{2}\right)-\frac{3 k_{u}^{4} v^{2} x_{L}^{4}}{4 \pi^{2} x_{S}^{2}} \log \frac{1+x_{L}^{2}}{x_{L}^{2}}+O\left(x_{S}^{-4}\right)
$$




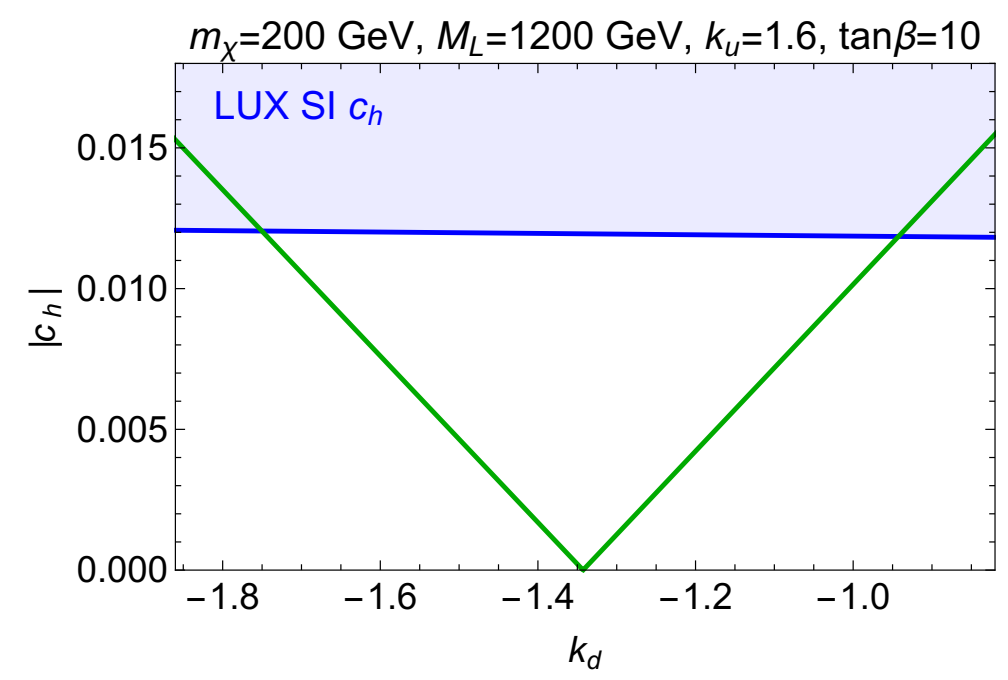

Figure 5. Values of the coupling $c_{h}$ while varying $k_{d}$ for a sample point of the parameter space. The values on the shaded area are excluded by LUX.

As noted in the previous subsection, the Higgs mass in this limit to leading order does not depend at all on the DM mass $M_{S}$. So the Higgs mass constraint to leading order in the mostly-singlet regime becomes a constraint on $k_{u}$ and $m / M_{L}$. For example, according to (5.3), in order to achieve $\delta m_{h}^{2}=3500 \mathrm{GeV}^{2}$ for $k_{u}=1.6$, we need $x_{L} \approx 1$.

\section{DM annihilation in the mostly-singlet regime}

An attractive feature of WIMP dark matter is its potential to naturally explain the observed relic abundance via the thermal freeze-out mechanism. Following the usual procedure (see e.g. the classic review [55]), we have

$$
\Omega_{\mathrm{DM}} h^{2} \approx 9.2 \times 10^{-12} \mathrm{GeV}^{-2} \times\left(\int_{x_{f}}^{\infty} d x \frac{\left\langle\sigma v_{\chi}\right\rangle}{x^{2}}\right)^{-1}
$$

The integral over $x$ takes into account annihilation after freeze-out, and $x_{f}=m_{\chi} / T_{f} \approx 25$ parametrizes the freeze-out temperature. $\left\langle\sigma v_{\chi}\right\rangle$ is the thermally-averaged DM annihilation cross section $\chi \chi \rightarrow X Y$, summed over all final states $X$ and $Y$. This is usually expanded in the small velocity limit:

$$
\sigma_{X Y} v_{\chi}=r_{X Y}\left(a_{X Y}+b_{X Y} v_{\chi}^{2}+O\left(v_{\chi}^{4}\right)\right)
$$

where $r_{X Y} \equiv \sqrt{1-\left(m_{X}+m_{Y}\right)^{2} / 4 m_{\chi}^{2}}$ is a kinematic phase space factor. At the time of freeze-out, the DM relative velocity is typically $v_{\chi}^{2} \sim 0.1$. Therefore, the annihilation cross section is generally controlled by the $s$-wave contributions $a_{X Y}$, unless they are suppressed for some reason.

In our model, the dark matter has many interactions and annihilation channels that should all be considered in full generality. As described in the introduction, for numerical 
calculations we use micrOMEGAs 4.1.8 [47] source code generated by SARAH 4.5.8 [44] to accurately take these into account. However in the mostly singlet limit that we are interested in, the cross sections simplify and we can have an analytic understanding of the behaviour of our model. We will assume that DM is lighter than all MSSM superpartners except possibly the Higgsinos, which are forced to be light $\mu \sim v$ by naturalness. In this case, the freeze-out process happens only through annihilation to SM particles and the Higgsinos. Including the Higgsinos in the story is a major difference from simplified-modelanalyses of singlet-doublet dark matter, which generally just add the singlet and doublets to the SM. As we will see, the Higgsinos can be a major part of the DM annihilation in the early universe.

The full cross sections are too complicated to print here. Instead, we will expand in the mostly-singlet limit $M_{S}<M_{L}, v \ll M_{L}, M_{L}-M_{S}$ with the further assumption that $v \ll M_{S}$. This suffices for our purposes and results in relatively simple expressions. (One exception is the tree-level, $s$-wave $t \bar{t}$ cross section in the next subsection, for which we can write down an extremely simple exact expression in terms of $c_{Z}$.)

\subsection{DM annihilation to fermions}

The fermions have $s$-wave contributions

$$
\begin{aligned}
a_{f \bar{f}} & =\frac{3 k_{u}^{4}}{32 \pi} \frac{m_{f}^{2}}{M_{L}^{4}\left(1-\epsilon^{2}\right)^{2}} \\
a_{\psi_{H} \psi_{H}} & =\frac{\left(k_{d}^{2}+k_{u}^{2}\right)^{2}}{16 \pi} \frac{\mu^{2}}{M_{L}^{4}\left(1+\epsilon^{2}+x_{L}^{2}\right)^{2}}
\end{aligned}
$$

where $\epsilon \equiv M_{S} / M_{L}$, and $x_{L} \equiv m / M_{L}$ was defined in section 4 . In the second line, we have summed over the various Higgsino final states including both neutralinos and charginos, assuming a pure MSSM Higgsino (i.e. $M_{1,2}$ decoupled). The fermion $b$ coefficients are always subdominant (suppressed by both $v_{\chi}^{2}$ and $v^{2} / M_{L}^{2}$ ), so we have not included them here.

The fermion cross sections are all suppressed by the square of the fermion mass, so $t \bar{t}$ and Higgsinos are the dominant channels. This is the famous $s$-wave helicity suppression of DM annihilation to fermion pairs.

Although $t \bar{t}$ and Higgsinos are parametrically similar, their diagrammatic origin is entirely different. The former (latter) arise from $s$-channel $Z$ ( $t$-channel superpartner) exchange. As a result, the Higgsinos are suppressed by the soft mass $m$. For $k_{u}=1.6$, we saw in section 5 that we need $x_{L} \approx 1$ for $m_{h}=125 \mathrm{GeV}$, so the suppression is not large. Also, $\mu$ is constrained to be $\lesssim 300 \mathrm{GeV}$ by naturalness. So all in all, the Higgsino contribution ends up generally of the same order or smaller than $t \bar{t}$.

The fact that the SM fermions all arise from $s$-channel $Z$ diagrams means that they have a simple exact expression beyond the small $v$ approximation:

$$
a_{f \bar{f}}=c_{Z}^{2} \frac{3 y_{f}^{2}}{4 \pi m_{Z}^{2}}
$$

In other words, $c_{Z}$ controls both the SD direct detection cross section and the annihilation to $t \bar{t}$. Therefore, we expect to see a fairly direct correlation between the SD direct detection limits and the relic density constraint. 


\subsection{DM annihilation to bosons}

Meanwhile the diboson cross sections are all $p$-wave to leading order:

$$
\begin{aligned}
b_{h h}=b_{Z Z} & =\frac{k_{u}^{4}}{384 \pi} \frac{\epsilon^{2}\left(3+2 \epsilon^{2}+3 \epsilon^{4}\right)}{M_{L}^{2}\left(1+\epsilon^{2}\right)^{4}} \\
b_{h Z} & =\frac{k_{u}^{4}}{96 \pi} \frac{\epsilon^{2}}{M_{L}^{2}\left(1+\epsilon^{2}\right)^{2}} \\
b_{W W} & =2 b_{h h}+b_{h Z}
\end{aligned}
$$

Here we took $\tan \beta \rightarrow \infty$ for simplicity; we checked that the $1 / \tan \beta$ corrections are irrelevant. The $s$-wave contributions are suppressed by $v^{4} / M_{L}^{4}$ so they are always subdominant to the $p$-wave contributions shown here.

Clearly, the diboson cross sections exhibit some interesting features. They are nonvanishing even in the $v \rightarrow 0$ limit, so they can be understood as a consequence of $\mathrm{SU}(2)_{L} \times$ $\mathrm{U}(1)_{Y}$ symmetry. These tree-level cross sections arise entirely due to the longitudinal components of the $W^{ \pm}$and $Z$ bosons, which by the Goldstone equivalence theorem are also equivalent to the charged and neutral Goldstones $G^{ \pm}$and $G^{0}$ respectively. Under a $\mathrm{U}(1)_{Y}$ rotation, $h \rightarrow G^{0}$ and $G^{0} \rightarrow-h$, while under an $\mathrm{SU}(2)_{L}$ rotation, $W^{ \pm} \rightarrow h \pm i G^{0}$. This explains both relations in (6.5).

Comparing $t \bar{t}$ and Higgsinos to the total diboson cross section, we see that parametrically the latter can be larger than the former, for sufficiently large $M_{L}$. However the cross over point is generally at very large $M_{L}$ and $M_{S}$. For instance, for $\epsilon=1 / 2$ and $x_{L}=1$, we find the cross over to be in the range $M_{L} \sim 2.7-3.6 \mathrm{TeV}$ for $\mu \sim 100-300 \mathrm{GeV}$. This is well beyond the naturalness-motivated part of the parameter space that we are focusing on in this paper. Therefore we conclude that the total $\sigma v_{\chi}$ is always dominated by $t \bar{t}$ and Higgsinos, and dibosons are always a subdominant part of it.

\subsection{Total annihilation cross section}

We have shown analytically that the relic density is dominated by $s$-wave annihilation to $t \bar{t}$ and Higgsinos (assuming of course that the DM is above the respective thresholds):

$$
\sigma v_{\chi} \approx a_{t \bar{t}}+a_{\psi_{H} \psi_{H}}=\frac{3 k_{u}^{4}}{32 \pi} \frac{m_{f}^{2}}{M_{L}^{4}\left(1-\epsilon^{2}\right)^{2}}+\frac{\left(k_{d}^{2}+k_{u}^{2}\right)^{2}}{16 \pi} \frac{\mu^{2}}{M_{L}^{4}\left(1+\epsilon^{2}+x_{L}^{2}\right)^{2}}
$$

A plot comparing our analytics to micrOMEGAs is shown in figure 6 for fixed choices of the parameters; we see there is excellent agreement across the entire range of relevant DM masses. We confirm that the dibosons are never more than $\sim 10 \%$ of the relic density across the entire parameter range of interest. Higgsinos and $t \bar{t}$ are comparable for $\mu \sim 300 \mathrm{GeV}$, while for $\mu \sim 100 \mathrm{GeV}, t \bar{t}$ dominates, as expected from the $\mu$ dependence of the Higgsino cross section (6.3).

One very interesting consequence of (6.6) is that in the limit of large $M_{L}$, the DM mass drops out of the annihilation cross section. Furthermore, we have seen that we need $k_{d} \sim k_{u}$ for the blind spot, $x_{L} \approx 1$ for the Higgs mass, and $\mu \sim m_{t}$ for naturalness. Thus the WIMP miracle transforms from being a constraint on the WIMP mass to being 

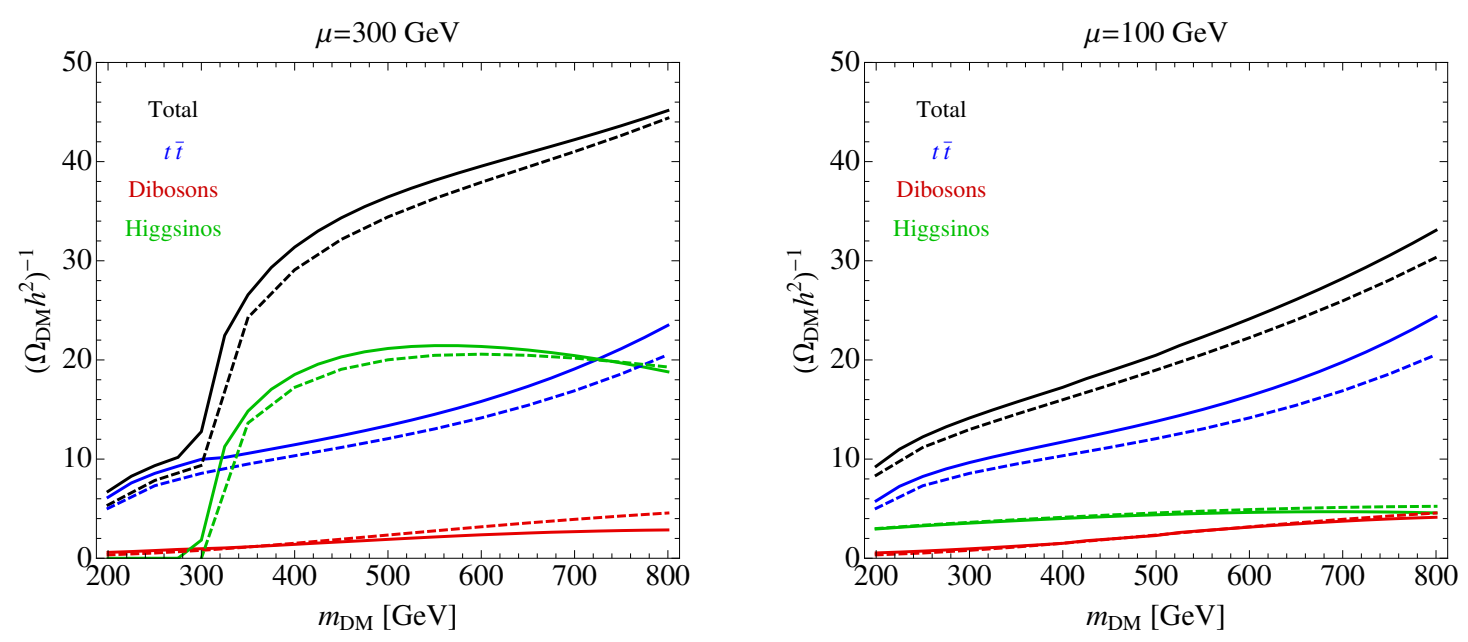

Figure 6. Inverses of the total relic abundance (black) as well as the individual contributions from $t \bar{t}$ (blue) and dibosons (red) as calculated numerically by micrOMEGAs 4.1.8 (solid) and the analytic equations (6.3) and (6.5) (dashed), for $M_{L}=1.2 \mathrm{TeV}, k_{u}=1.6, k_{d}=-1.5$ and $\tan \beta=10$.

a constraint on the mediator scale $M_{L}$ ! This helps to relieve the "WIMP little hierarchy problem", whereby the preference of the thermal relic constraint for TeV-scale WIMPs is in tension with naturalness. Comparing with (5.2), we also expect that the relic density constraint will essentially fix $c_{Z}$ to a unique value. We will confirm this in the next section with our full numerical scans and discuss its implications for SD direct detection.

\section{$7 \quad$ Putting it all together}

\subsection{Plots in the $M_{L}-M_{S}$ plane}

Having described the various individual components of the analysis of the model (direct detection, the Higgs mass, and the relic abundance), we will now combine them and describe how the different constraints interact to produce the viable parameter space of the model.

In figure 7 we show contour plots for numerical scans over the $\left(M_{L}, M_{S}\right)$ plane for fixed values of $k_{u}, k_{d}$ and $\mu$. We choose four sets of benchmark parameters: large coupling $\left(k_{u}=1.6, k_{d}=-1.5\right)$ and small coupling $\left(k_{u}=1.2, k_{d}=-1.5\right)$; and large $\mu(\mu=300 \mathrm{GeV})$ and small $\mu(\mu=100 \mathrm{GeV})$.

We see the impact of the direct detection limits on the parameter space of the model. ${ }^{7}$ The LUX SI and SD limits are strongest almost everywhere except a tiny sliver for large $M_{S}$ in the $k_{u}=1.2, k_{d}=-1.5$ case where IceCube has an impact. (Note that the LUX

\footnotetext{
${ }^{7}$ We have not overlaid indirect detection limits on this plot. As already mentioned in the introduction, for DM masses above $\sim 100 \mathrm{GeV}$, there are no constraints from Fermi [39] for points with the correct relic abundance. For points with $\Omega_{\mathrm{DM}}>0.12,\left\langle\sigma v_{\chi}\right\rangle$ would be even smaller and Fermi would not constrain any of these points either. Finally, for points with $\Omega_{\mathrm{DM}}<0.12$ there could be indirect detection constraints, but in this case the model should be completed by having multi-component DM and/or non-thermal processes, giving many possible new signatures. We leave a detailed analysis of these extensions of our model for future work.
} 

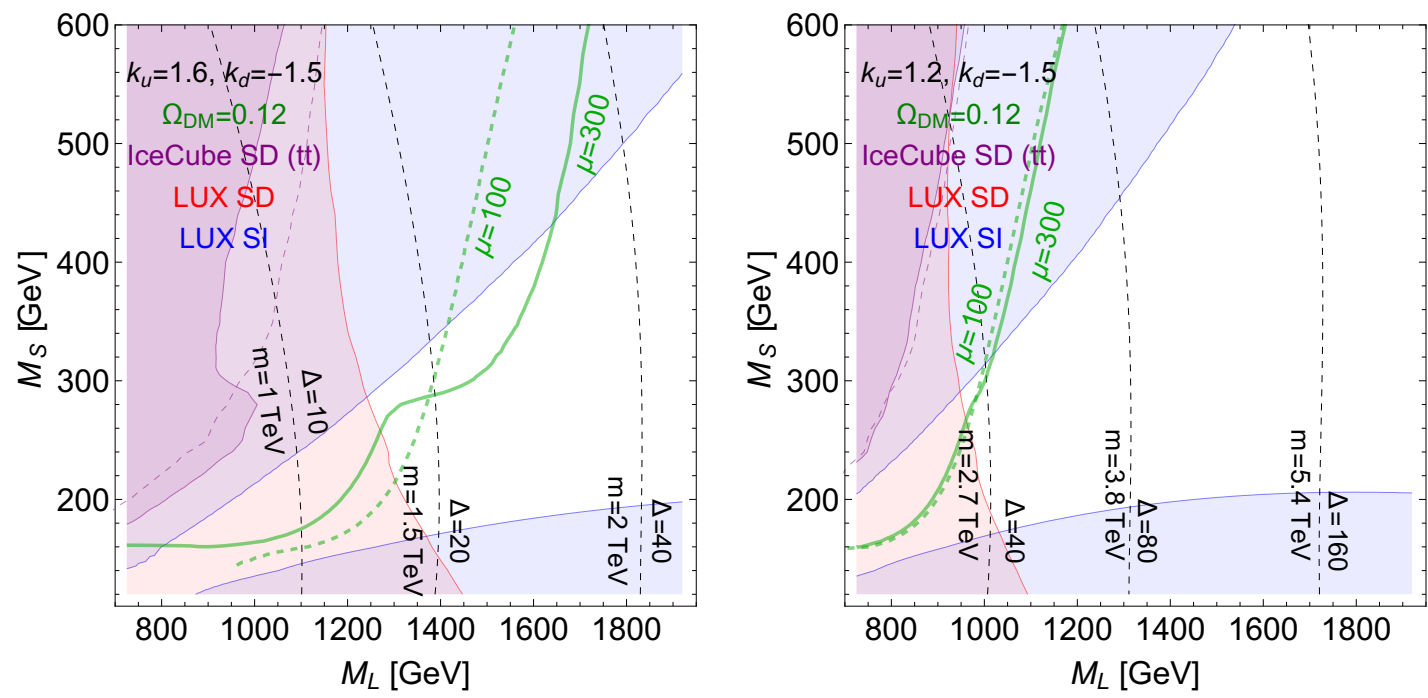

Figure 7. $M_{L}-M_{S}$ parameter space scan with micrOMEGAs 4.1 .8 [47] for $k_{u}=1.6, k_{d}=-1.5$ (left) and $k_{u}=1.2, k_{d}=-1.5$ (right), $\tan \beta=10$ and all MSSM soft masses and gauginos at $1 \mathrm{TeV}$. We show the exclusion regions from $\sigma^{\mathrm{SI}}$ in blue and $\sigma_{n}^{\mathrm{SD}}$ in red, both from LUX [35] and for $\sigma_{p}^{\mathrm{SD}}$ from IceCube $t \bar{t}$ with $\mu=300 \mathrm{GeV}(\mu=100 \mathrm{GeV})$ [36] in solid (dashed) purple. The dashed black lines are the fine-tuning contours for $\mu=300 \mathrm{GeV}$. The contour of $\Omega_{\mathrm{DM}}=0.12$ with $\mu=300 \mathrm{GeV}$ $(\mu=100 \mathrm{GeV})$ is in solid (dashed) green.

limits assume the singlet-doublet sector comprises all of the DM, regardless of whether it is thermal or not.) The SD (SI) limits primarily cover the lighter (heavier) DM mass region. The heavier DM region is ruled out because we are holding fixed $k_{d}$, so as one increases $m_{\chi}$ the blind spot cancellation shown in (5.2) becomes less effective.

For every point in the plane we numerically solved (using SPheno) the $m_{h}=125 \mathrm{GeV}$ constraint for the common soft mass $m$; these contours are shown in figure 7 along with their corresponding tuning. These contours are mostly vertical; as discussed in section 4, the soft mass and $\Delta$ depend primarily on $M_{L}$ since the dependence on $M_{S}$ drops out to leading order at large $M_{L}$.

Finally, we used micrOMEGAs to numerically solve the thermal relic density constraint $\Omega_{\mathrm{DM}}=0.12$ [56]; this fixes $M_{L}$ as a function of $M_{S}$ and these contours are shown in green for various choices of the parameters. Note the rapid increase in $M_{L}$ across the top and Higgsino thresholds. Here new $s$-wave annihilation channels open up, and so larger values of $M_{L}$ are needed to maintain the overall annihilation rate at the thermal relic value. This effect is more pronounced for larger values of $k_{u, d}$ and for larger values of $\mu$. Indeed, in section 6 we saw that the annihilation cross sections to $t \bar{t}$ and Higgsinos are enhanced for greater $k_{u, d}$, and the Higgsino cross section in particular is proportional to $\left(k_{d}^{2}+k_{u}^{2}\right)^{2} \mu^{2}$.

Since larger $M_{L}$ decreases direct detection cross sections, increasing $k_{u, d}$ and $\mu$ also increases the viable parameter space for thermal relic DM. The Higgsino channels in particular allow the model to survive the direct detection limits over a wider range of parameter space than would have been the case for non-supersymmetric singlet-doublet 


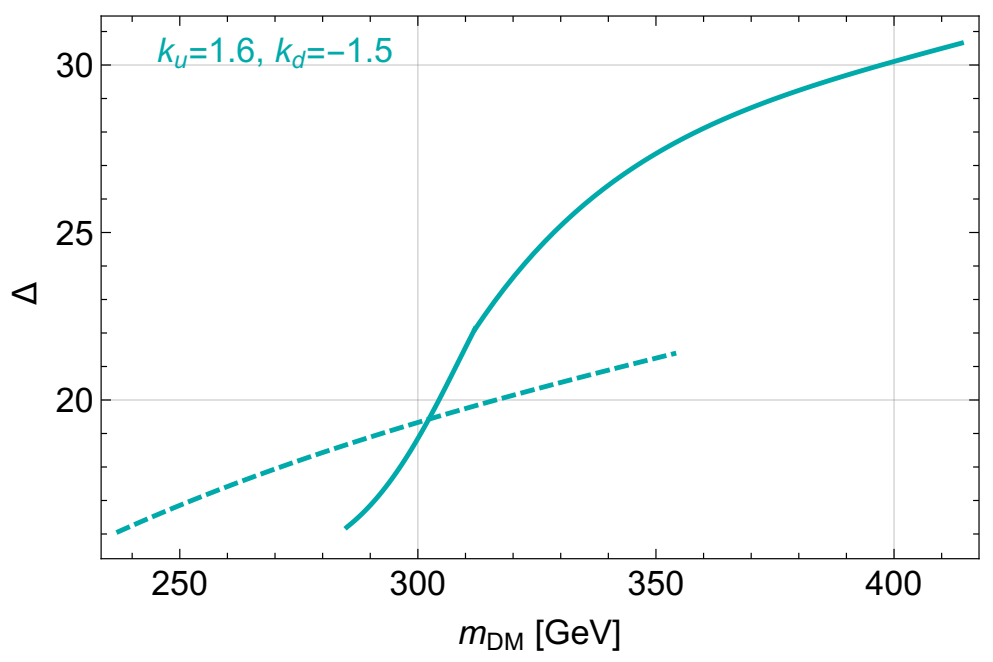

Figure 8. Fine-tuning for the right relic abundance contours of figure 7 (left) that are allowed by direct detection. We show the case for $\mu=300 \mathrm{GeV}$ (solid) and $\mu=100 \mathrm{GeV}$ (dashed).

DM. Figure 7 also shows that larger $k_{u, d}$ is better for fine-tuning, confirming our discussion in section 4 . The only potential drawback of the larger coupling choice is (as we will discuss in section 8.1) that the former has a lower Landau pole $\left(\Lambda \sim 10^{2} \mathrm{TeV}\right.$ vs. $\left.\Lambda \sim 10^{3} \mathrm{TeV}\right)$.

Away from the top and Higgsino thresholds, we see that the relic density contours are mostly vertical, meaning that the relic density constraint becomes a constraint primarily on $M_{L}$, once the other parameters $\left(k_{u}, k_{d}, m, \mu\right)$ are fixed, i.e. the WIMP DM mass drops out to leading order. This confirms our analytics in the previous section.

\subsection{Projecting onto the thermal relic contour}

Finally let us impose the relic density constraint $\Omega_{\mathrm{DM}}=0.12$ and see how various parameters vary along the green contours in figure 7 . In figure 8 we show the fine-tuning for the points with the correct relic abundance. It is remarkable that there are allowed regions of the parameter space with $\Delta \lesssim 20$, making this model much less tuned than the MSSM.

In figure 9 we show $c_{h}$ for the points of the parameter space that satisfy $\Omega_{\mathrm{DM}}=0.12$ (including both allowed and excluded points from direct detection limits). We see that varying $k_{d}$ we can move toward the blind spot and satisfy the SI direct detection bounds.

Similarly, in figure 10 we show $c_{Z}$ for the points of the parameter space with $\Omega_{\mathrm{DM}}=$ 0.12 (including both allowed and excluded points from direct detection limits). We can see that for $m_{\mathrm{DM}}>\mu$ contours of constant $\Omega_{\mathrm{DM}}$ have an approximately constant $c_{Z}$. This confirms the discussion based on analytics in section 6.3. Indeed, using (6.6) with the parameter choices here, we find that for $100 \mathrm{GeV} \leq \mu \leq 300 \mathrm{GeV}, c_{Z}$ ranges from $0.005 \lesssim c_{Z} \lesssim 0.008$. These values are clearly illustrated in figure 10 .

We conclude that $c_{Z}$ (and consequently $\sigma^{\mathrm{SD}}$ ) is basically fixed by the relic density constraint. Requiring $\chi$ to be all the dark matter leads to a nearly unique prediction for the SD cross section! Fortunately, as shown in figure 10, these values of $c_{Z}$ are still 

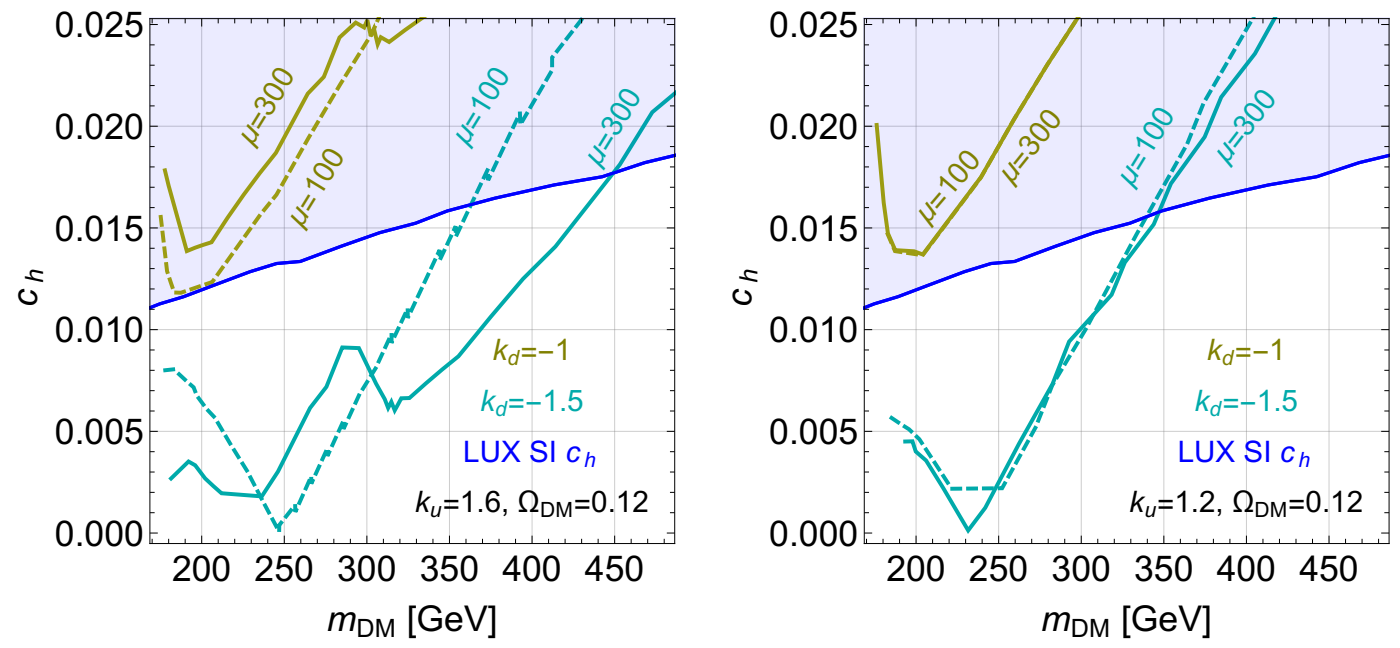

Figure 9. Values of the coupling $c_{h}$ for the points with $\Omega_{\mathrm{DM}}=0.12$ for different values of $k_{d}$. We show the values for $k_{u}=1.6$ (left) and $k_{u}=1.2$ (right). The exclusion region from $\sigma^{\mathrm{SI}}$ is in blue.
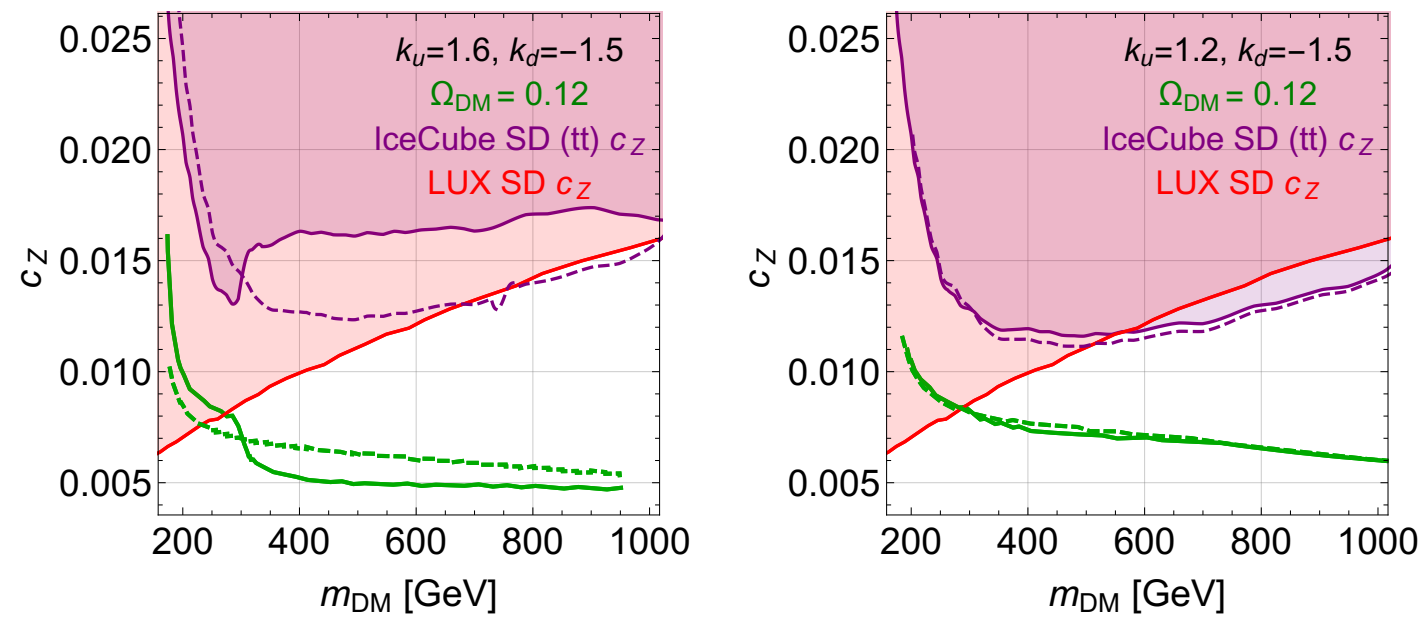

Figure 10. Values of the coupling $c_{Z}$ for the points with $\Omega_{\mathrm{DM}}=0.12$ for $\mu=300 \mathrm{GeV}(\mu=$ $100 \mathrm{GeV}$ ) in solid (dashed) green. The exclusion region from $\sigma_{n}^{\mathrm{SD}}$ is in red and from $\sigma_{p}^{\mathrm{SD}}$ with $\mu=300 \mathrm{GeV}(\mu=100 \mathrm{GeV})$ is in solid (dashed) purple.

allowed by the current direct detection experiments, IceCube in particular. ${ }^{8}$ With factor of 10-100 improvements in cross section expected from Xenon1T [37] and LZ [38], the next generation of DM direct detection experiments will be sensitive to essentially the entire parameter space of this model (assuming $\chi$ is a thermal relic and is all the DM). A discovery might be right around the corner!

\footnotetext{
${ }^{8}$ It is quite crucial that our DM annihilates almost exclusively to $t \bar{t}$ and Higgsinos. The IceCube bound on $t \bar{t}$ is by a factor of a few weaker than the $W^{+} W^{-}$cross section, and it saves the model from being already ruled out. The case of Higgsinos decaying to Higgs and gravitino does not introduce any new regions of parameter space that were not already ruled out by LUX [35] in figure 7. (Also, as the DM annihilation to Higgsinos arises from t-channel superpartner exchange, there would be no constraints on $c_{Z}$ from this channel.)
} 


\section{Outlook}

In this section we briefly discuss the UV behavior of the model (in particular the Landau poles) and the potential sensitivity from LHC Run II. Finally we conclude with some thoughts on future directions.

\subsection{UV considerations}

So far we have been exploring our model at the EW scale and have identified the interesting parts of the parameter space around $k_{u} \sim k_{d} \sim 1.2-1.6$. Here we want to examine the UV consequences of such large Yukawa couplings and comment on possible solutions to the Landau pole problem.

Let's focus on the most important couplings, $\left(g_{1}, g_{2}, g_{3}, y_{t}, k_{u}, k_{d}\right)$ and neglect the effect of the other couplings in finding the scale of Landau poles. Starting from one loop beta functions above the scale of the new fields (including spectator color triplets for unification) we have

$$
\begin{aligned}
& \beta_{g_{i}}=\frac{b_{i}}{16 \pi^{2}} g_{i}^{3} \quad\left(b_{1}, b_{2}, b_{3}\right)=\left(\frac{36}{5}, 2,-2\right) \\
& \beta_{k_{u}}=\frac{k_{u}}{16 \pi^{2}}\left(2 k_{d}^{2}+4 k_{u}^{2}+3 y_{t}^{2}-\frac{3}{5} g_{1}^{2}-3 g_{2}^{2}\right) \\
& \beta_{k_{d}}=\frac{k_{d}}{16 \pi^{2}}\left(4 k_{d}^{2}+2 k_{u}^{2}-\frac{3}{5} g_{1}^{2}-3 g_{2}^{2}\right) \\
& \beta_{y_{t}}=\frac{y_{t}}{16 \pi^{2}}\left(6 y_{t}^{2}+k_{u}^{2}-\frac{16 g_{3}^{2}}{3}-\frac{13}{15} g_{1}^{2}-3 g_{2}^{2}\right)
\end{aligned}
$$

Solving the RGE's of our model numerically, we can find the lowest scale at which one of the couplings hits its Landau pole. In figure 11 we show this scale as a function of the Yukawa couplings at $1 \mathrm{TeV}$.

Note that for $k_{u} \lesssim 2$ (as we have considered in this work), the Landau poles are above $100 \mathrm{TeV}$. Now we might ask: how can we understand physics above the Landau pole scale, or how can we postpone it to higher energies e.g. the GUT scale? One idea is to use non-Abelian gauge interactions for the new sector to reduce the beta functions of the Yukawa couplings: if we include multiple copies of $S, L, \bar{L}$ and charge them under a non-Abelian gauge group, the corresponding gauge coupling appears with negative sign in the beta function of $k_{u}, k_{d}$ (see e.g. [32] for a recent implementation of this idea). As $S$ is Majorana, we need $S$ to be in a real representation of the new gauge group. A simple example is when the gauge group is $\mathrm{SO}(N)$ and $S$ is in the fundamental representation. Another possibility might be to match our model to the magnetic side of a Seiberg duality and interpret physics above the scale of the Landau pole by the electric theory. It will be interesting to explore these ideas further in the future.

\subsection{LHC phenomenology}

In addition to direct detection experiments, DM models are also probed by the LHC. In principle, monojet+MET [40, 41] and monophoton+MET [42, 43] searches for direct DM production could be sensitive to our model. Since quarks and gluons only talk to $\chi$ through 


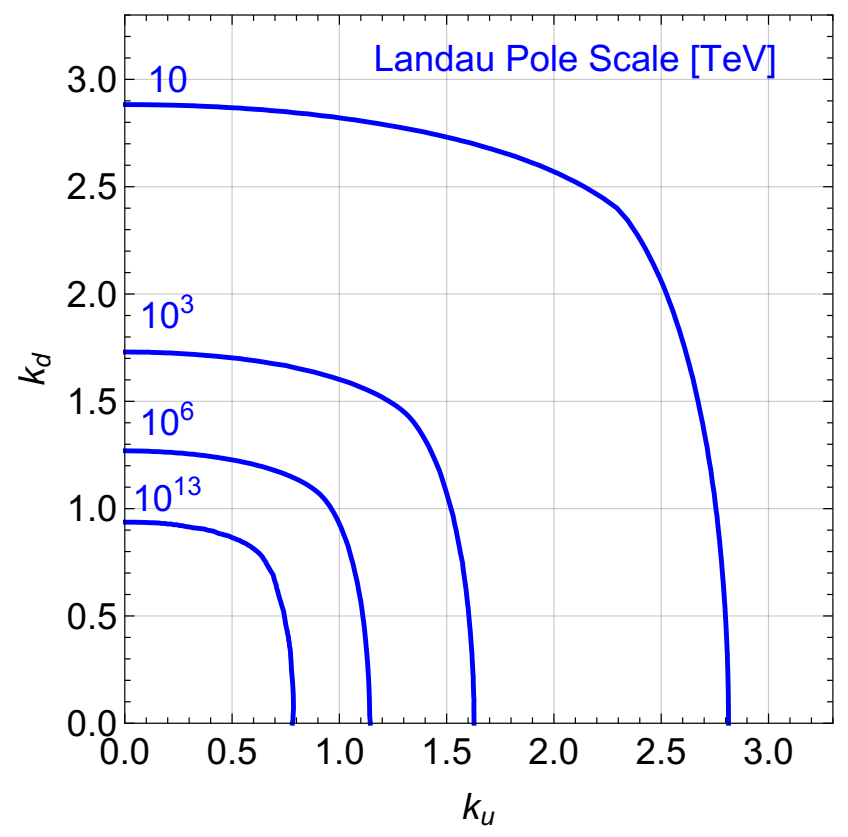

Figure 11. Scale of Landau poles with one-loop RGE's in terms of $k_{d}, k_{u}$ at the IR scale. We are assuming for each point on the plot that $k_{u}$ and $k_{d}$ are given at $\Lambda_{\mathrm{IR}}=1 \mathrm{TeV}$.

$s$-channel diagrams involving $Z$ 's and Higgses, these searches constrain the same $c_{Z}$ and $c_{h}$ couplings as direct detection. However, these constraints are weaker by several orders of magnitude than those from direct detection under the assumption that our DM candidate $\chi$ is all of the relic density, for the mass range we consider. See e.g. $[57,58]$ for a recent discussion in terms of simplified DM models.

Instead, let us briefly consider mono $(h, W, Z)+$ MET. This can occur in our model through production of $\chi_{1} \chi_{2,3}^{0}$ and $\chi_{1} \chi^{ \pm}$and subsequent decay of the (mostly-doublet) $\chi_{2,3}^{0}$ and $\chi^{ \pm}$. (Here all $\chi^{\prime}$ 's refer to dark sector fermion mass eigenstates, not MSSM neutralinos and charginos.) A full treatment including estimation of SM backgrounds, detector acceptances, etc. is beyond the scope of this work. Here we will just present the raw production cross sections in our model.

Diagrams contributing to mono-Higgs/ $W+$ MET are shown in figure 12 (mono$Z+\mathrm{MET}$ is the same as mono-Higgs with the final state Higgs replaced by $Z$ ). Note that we have included the one-loop gluon fusion diagram. ${ }^{9}$ Because of the large, $O(1)$ Yukawas $k_{u}, k_{d}$ in this model, this contribution can be as much as $60 \%$ of the total $\chi_{1} \chi_{2,3}^{0}$ cross section. We calculated the gluon fusion contribution analytically, and the tree level contributions both analytically and with MadGraph5 [59] using the model file generated by SARAH and the spectrum files generated by SPheno. More details on the analytics are given in appendix C. In both cases, we used the NNPDF2.3 [60] PDF set. Figure 13 shows the sum of tree level contributions and gluon fusion along the $\Omega_{\mathrm{DM}}=0.12$ contour. We see that LHC13 will ultimately be able to probe the small mass region. Of course, if $\chi$ is all of the dark matter, then direct detection experiments will discover the model first. In that

\footnotetext{
${ }^{9}$ We thank Matt Reece for bringing this to our attention.
} 

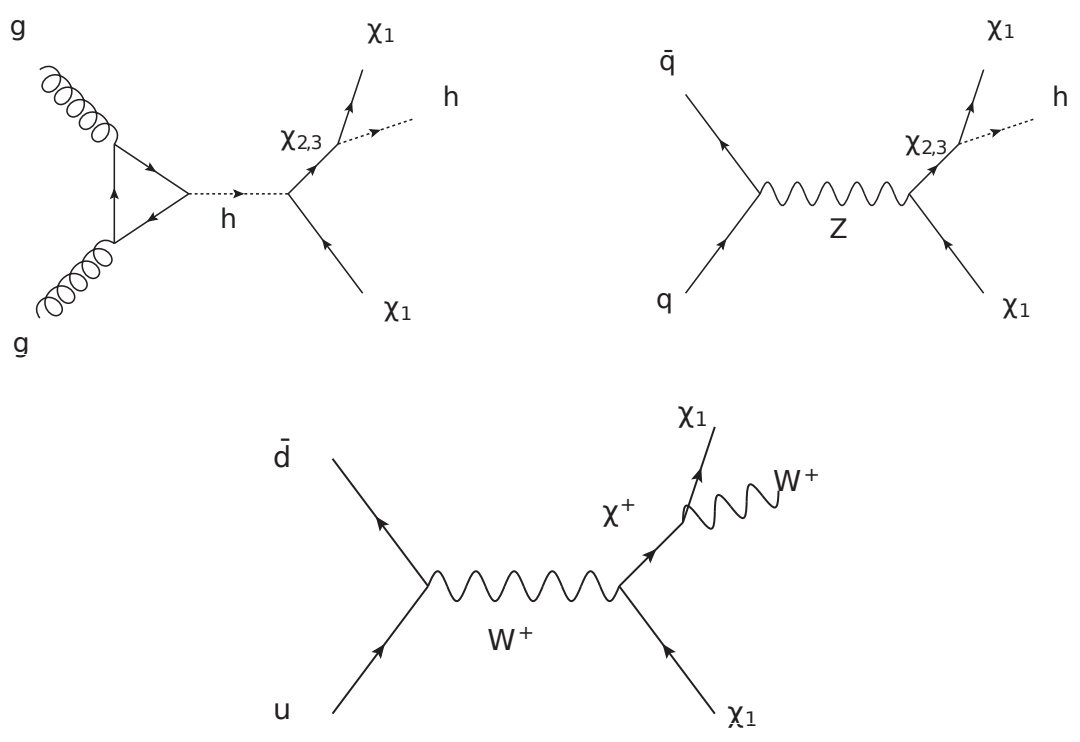

Figure 12. Diagrams contributing to mono-Higgs/Z/W+MET in our model.
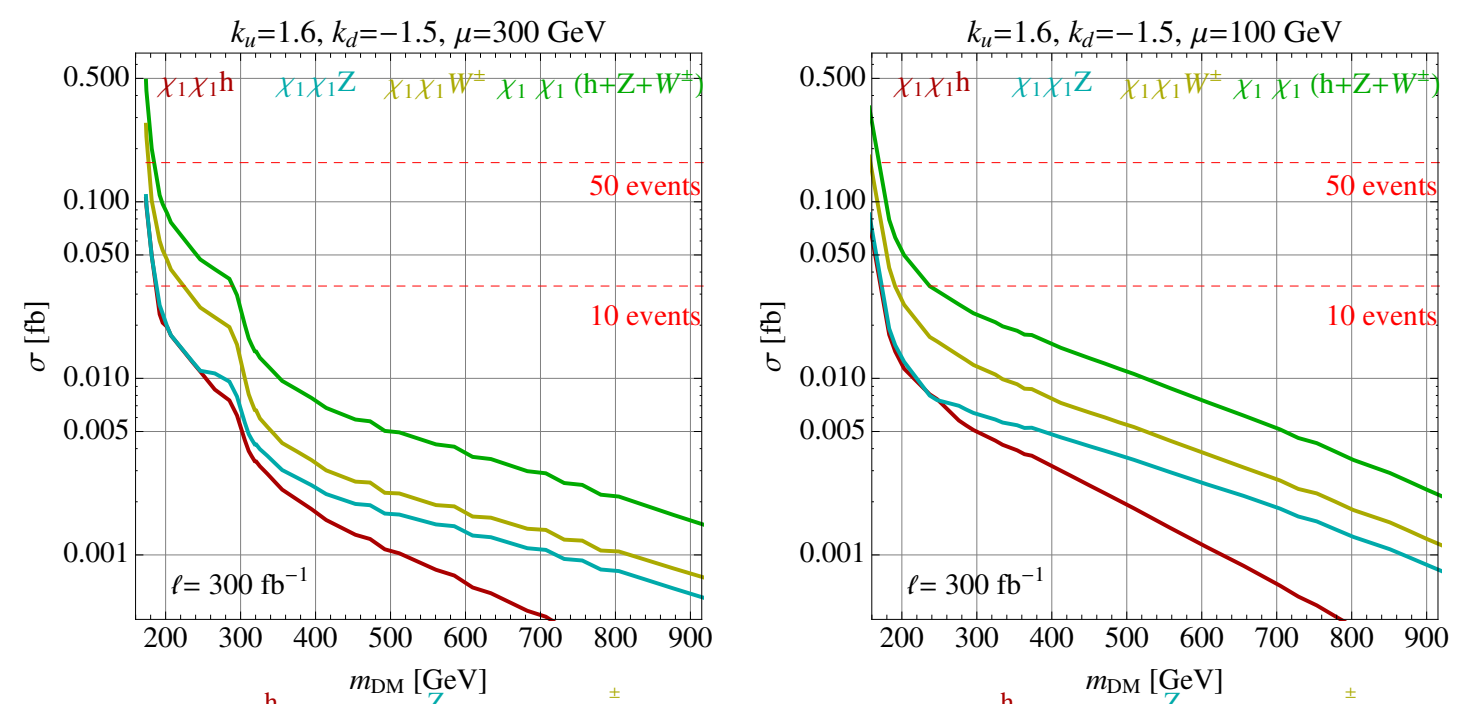

Figure 13. Final state cross sections for LHC13 for the points with $\Omega_{\mathrm{DM}}=0.12$ from figure 7 (left), $\tan \beta=10$ and all superpartners decoupled. As these points depend on the annihilation cross section to Higgsinos, we specify the value of $\mu=300 \mathrm{GeV}$ (left) and $\mu=100 \mathrm{GeV}$ (right). We show in green the total cross section (adding $h, Z$ and $W^{ \pm}$channels). For the $W$ cross section we are showing the sum of $W^{+}$and $W^{-}$final states.

case, the LHC will only be useful as a post-discovery confirmation of the model. However, since the LHC is producing $\chi$ directly, it does not depend on the relic density. Therefore if our dark sector is only one component of $\Omega_{\mathrm{DM}}$, the direct detection limits could be greatly relaxed while the $\mathrm{LHC}$ would remain sensitive. 


\subsection{Future directions}

The work presented in this paper is a simple realization of a general idea: economically extending the MSSM with a single sector that provides both thermal WIMP dark matter and the $125 \mathrm{GeV}$ Higgs mass. Here we took this sector to be a singlet and a pair of doublets, but one could easily imagine many other possibilities. For instance, very popular ideas for lifting the Higgs mass include the NMSSM (see e.g. [61] for a review and original references) and non-decoupling D-terms [62, 63]. While dark matter in the NMSSM is a well-studied topic, it would be very interesting to try to connect non-decoupling D-terms to dark matter.

Even within the context of our specific singlet-doublet model, there are many interesting open questions. In this work we made some simplifying assumptions in our analysis, and it would be interesting to explore the consequences of relaxing these assumptions. For example, we took all model parameters to be real, but in general there is one physical CP-violating phase. The effect of this phase on direct detection and annihilation cross sections can qualitatively change the model's behavior. Furthermore, we took all the soft mass-squareds to be positive to increase the Higgs mass. One might wonder how the phenomenology of the model would change if one of the soft masses is negative and the DM is a scalar instead of a fermion. We also assumed negligible $A$-terms in the dark sector. By analogy to stops, having substantial $A$-terms can help in raising the Higgs mass, see e.g. [19]. This could allow for smaller $k_{u}, k_{d}$ and open up more of the parameter space. Additionally, we focused on dark matter above $\sim 100 \mathrm{GeV}$. It could be interesting to study the phenomenology of the model for lighter dark matter masses. In particular the annihilations through the Higgs and $Z$ resonances could be large enough while still having suppressed direct detection signals. Finally, one could relax the assumption that $\chi$ is thermal and is all of the DM, and consider non-thermal relics or multi-component DM scenarios. All of these directions will become especially well-motivated if nothing is discovered at the next round of direct detection experiments, as discussed in section 7 .

There are also many interesting model-building directions in the UV. For example, enlarging the dark sector to accommodate a non-Abelian gauge symmetry could have potentially interesting consequences. As noted in section 8.1, this may help postpone the Landau pole of the Yukawa couplings, and the new gauge interactions could play an important role in the dynamics of the dark sector. Additionally we have two supersymmetric masses $M_{L}$ and $M_{S}$ at the electroweak scale. Perhaps the same dynamics that generates $\mu$ in the MSSM is responsible for generating these masses as well.

\section{Acknowledgments}

We thank Kyu Jung Bae, Matt Buckley, John Paul Chou, Eva Halkiadakis, Ryuichiro Kitano, Stephen Martin, Natsumi Nagata, Matt Reece, Alessandro Strumia and Scott Thomas for helpful discussions. We are especially grateful to Florian Staub for his indispensable help setting up the model in SARAH. We are also grateful to Natsumi Nagata and Florian Staub for comments on the manuscript. This work was supported by DOE grant DE-SC0013678. 


\begin{tabular}{|c|c|c|c|c|c|c|}
\hline & $\Delta_{u}$ & $\Delta_{d}$ & $\Delta_{s}$ & $f_{u}$ & $f_{d}$ & $f_{s}$ \\
\hline$p$ & 0.842 & -0.427 & -0.085 & 0.0153 & 0.0191 & 0.0447 \\
\hline$n$ & -0.427 & 0.842 & -0.085 & 0.011 & 0.0273 & 0.0447 \\
\hline
\end{tabular}

Table 2. Nucleon quark form factors.

\section{A Connecting model parameters to DD cross sections}

In this appendix, we will review how to relate the SI and SD DM-nucleon cross sections to the couplings $\xi_{q}^{\mathrm{SI}}$ and $\xi_{q}^{\mathrm{SD}}$ appearing in the effective Lagrangian (3.1). To check our results we verify that by calculating SI and SD cross sections analytically, we get the same result as the one we get from micrOMEGAs.

Following [55], the SI and SD cross sections are

$$
\sigma_{p, n}^{\mathrm{SI}}=\frac{x m_{r}^{2}}{\pi} f_{p, n}^{2}, \quad \sigma_{p, n}^{\mathrm{SD}}=\frac{3 x m_{r}^{2}}{\pi} a_{p, n}^{2}
$$

where $x=4$ for Majorana ( $x=1$ for Dirac) fermions, $m_{r}$ is the reduced mass of the DM-nucleon system, and $a_{p, n}^{\mathrm{SD}}, f_{p, n}^{\mathrm{SI}}$ are the effective DM-nucleon couplings:

$$
\begin{aligned}
f_{p, n} & =\sum_{q=u, d, s} \xi_{q}^{\mathrm{SI}} f_{q}^{p, n} \frac{m_{p, n}}{m_{q}}+\frac{2}{27}\left(1-\sum_{q=u, d, s} f_{q}^{p, n}\right) \sum_{q=c, b, t} \xi_{q}^{\mathrm{SI}} \frac{m_{p, n}}{m_{q}} \\
a_{p, n} & =\sum_{q=u, d, s} \xi_{q}^{\mathrm{SD}} \Delta_{q}^{p, n}
\end{aligned}
$$

Here $m_{q}$ is the quark mass, $f_{q}^{p, n}$, and $\Delta_{q}$ are hadronic parameters calculated for example by lattice simulations in QCD. We use the values in table 2 according to [64]. The difference between SI cross sections for proton and neutron is negligible as the main contribution comes from $f_{s}$ which is the same in both cases.

In figure 14, we compare our analytic cross sections to micrOMEGAs. We see that the agreement is excellent.

\section{B Validating SPheno one loop Higgs mass}

In this appendix we validate the contributions to the Higgs mass from the dark sector as calculated by SPheno against the analytic one-loop calculation through the ColemanWeinberg $(\mathrm{CW})$ potential. We consider the simplified one-loop CW result from (4.1), where we ignored $g_{1,2}$ and $\mu$ and took the $\tan \beta \rightarrow \infty$ limit. We will show that both one-loop and two-loop results from SPheno match quite well with our analytical result. As SPheno outputs the total Higgs mass and not just the contributions from the dark sector, we extract these contributions as follows:

$$
\delta m_{h}^{2}=m_{h}^{2}-\left.m_{h}^{2}\right|_{\mathrm{MSSM}}
$$

where $\left.m_{h}^{2}\right|_{\text {MSSM }}$ is the contribution to $m_{h}^{2}$ from the MSSM with superpartners at $1 \mathrm{TeV}$. 

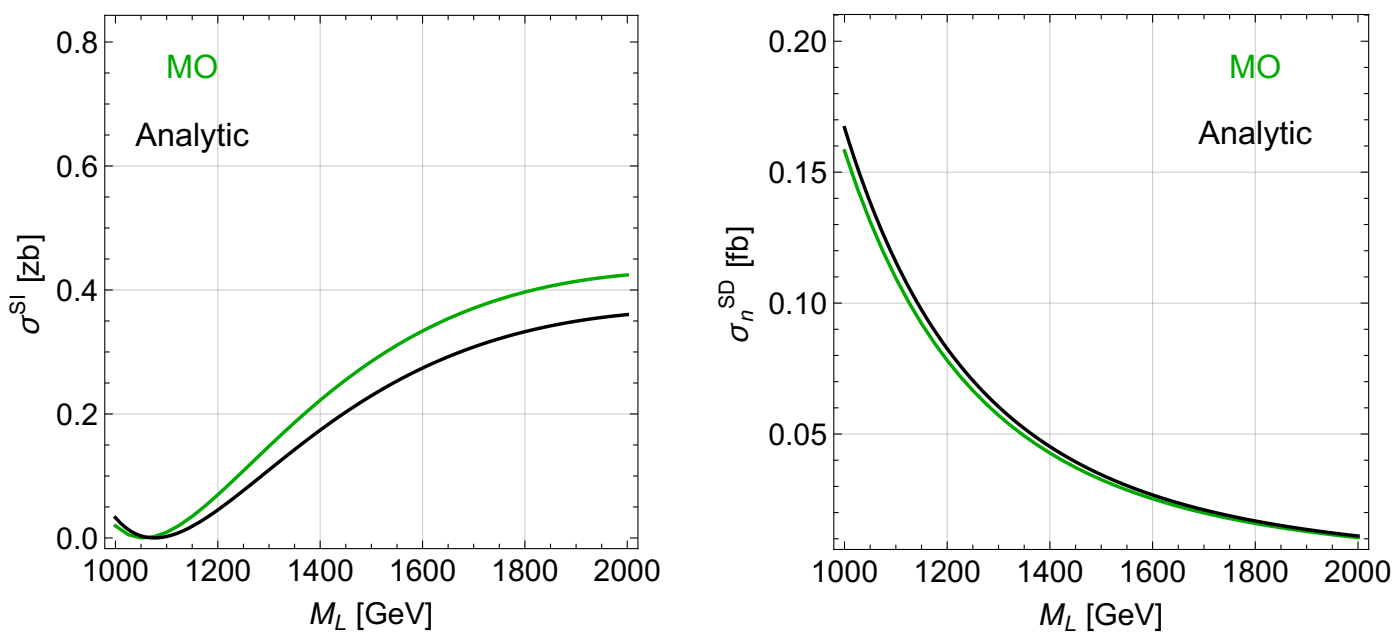

Figure 14. Comparing (A.1) with micrOMEGAs 4.1 .8 for $M_{S}=200 \mathrm{GeV}, k_{u}=1.6, k_{d}=-1.5$.
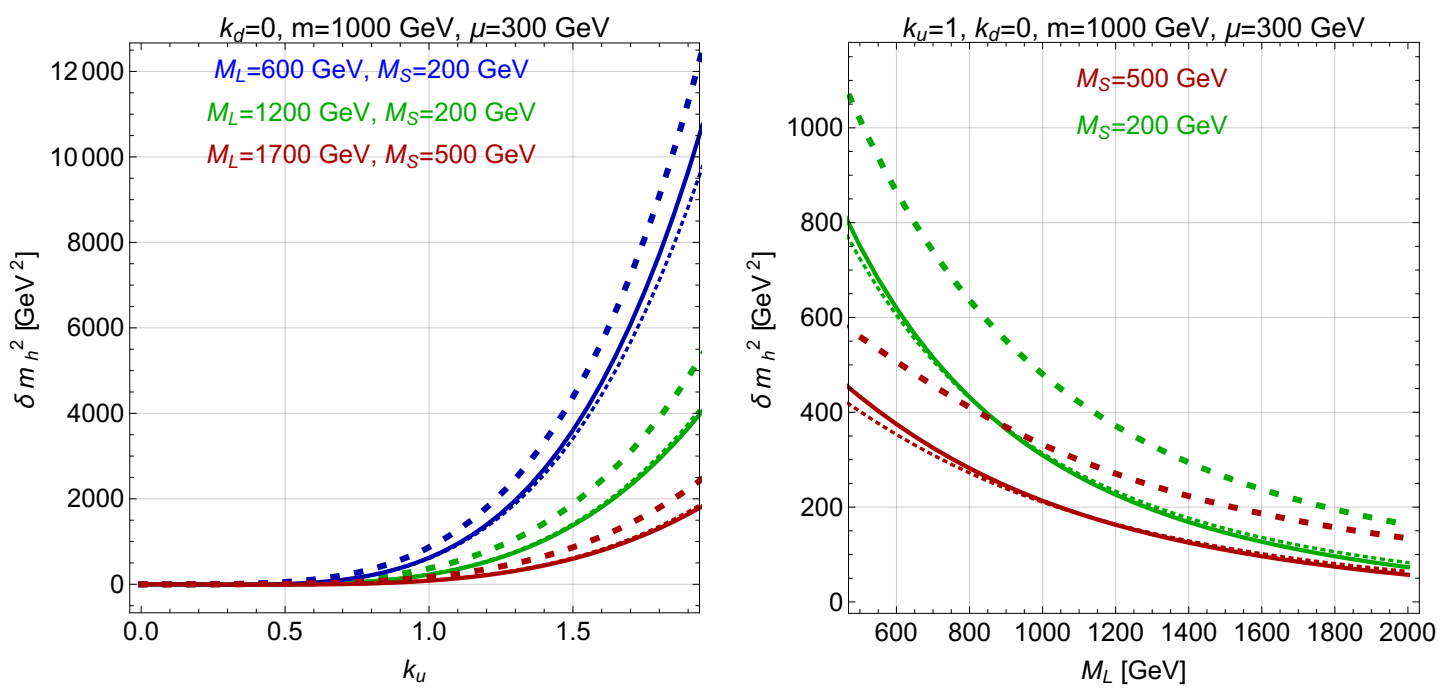

Figure 15. Thick dashed: analytical one-loop result (4.1). Dotted: SPheno one-loop. Solid: SPheno two-loop.

Since the Higgs mass depends primarily on $k_{u}, M_{L}, M_{S}$, we will demonstrate here that SPheno and our CW calculation agree well as these parameters are varied. From (4.1) we expect $\delta m_{h}^{2} \sim k_{u}^{4}$. As we can see in figure 15 (left), SPheno confirms this behavior. After fixing the $k_{u}$ dependence, we need to check that our analytical equations and SPheno match as we change $M_{L}$ and $M_{S}$. That is shown in figure 15 (right) for two values of $M_{S}$ as we scan over $M_{L}$.

\section{LHC cross section analytics}

Here we will provide some analytic details for the calculation of the $p p \rightarrow \chi_{1} \chi_{2,3}$ and $p p \rightarrow \chi_{1} \chi^{ \pm}$LHC cross sections useful for section 8.2. The former receives contributions 
from both tree-level $Z$ 's with $q \bar{q}$ initial state, as well as one-loop gluon fusion. The latter comes from tree-level $W^{ \pm}$'s with $q \bar{q}^{\prime}$ initial state.

The tree-level, quark-initiated cross sections are given by:

$$
\begin{aligned}
\sigma\left(q \bar{q} \rightarrow \chi_{1} \chi_{i}\right)= & \frac{g_{2}^{4}\left|\vec{p}_{f}\right|}{144 \pi c_{W}^{4} S^{3 / 2}}\left(c_{Z q_{L} q_{L}}^{2}+c_{Z q_{R} q_{R}}^{2}\right) \\
& \times\left(\left(\operatorname{Re} R_{i}\right)^{2} f_{Z}\left(S, m_{1}, m_{i}\right)+\left(\operatorname{Im} R_{i}\right)^{2} f_{Z}\left(S, m_{1},-m_{i}\right)\right) \\
\sigma\left(q \bar{q}^{\prime} \rightarrow \chi_{1} \chi^{+}\right)= & \frac{g_{2}^{4}\left|\overrightarrow{p_{f}}\right|}{576 \pi S^{3 / 2}}\left(f_{W}\left(S, m_{1}, m_{+}\right)\left|R_{+}\right|^{2}+f_{W}\left(S, m_{1},-m_{+}\right)\left|R_{-}\right|^{2}\right)
\end{aligned}
$$

where

$$
\begin{aligned}
R_{i} & =\left(U_{1,2}^{*} U_{i, 2}-U_{1,3}^{*} U_{i, 3}\right) \\
R_{ \pm} & =U_{1,2} \pm U_{1,3}^{*} \\
f_{A}(S, x, y) & =\frac{\left(S-(x+y)^{2}\right)\left(S+\frac{(x-y)^{2}}{2}\right)}{\left(S-m_{A}^{2}\right)^{2}}
\end{aligned}
$$

and

$$
c_{Z \bar{u}_{L} u_{L}}=\frac{1}{2}-\frac{2}{3} s_{W}^{2}, \quad c_{Z \bar{d}_{L} d_{L}}=-\frac{1}{2}+\frac{1}{3} s_{W}^{2}, \quad c_{Z \bar{u}_{R} u_{R}}=\frac{2}{3} s_{W}^{2}, \quad c_{Z \bar{d}_{R} d_{R}}=-\frac{1}{3} s_{W}^{2}
$$

The parton level gluon fusion cross section (as can be calculated e.g. using [50]) is

$$
\sigma\left(g g \rightarrow \chi_{1} \chi_{i}\right)=\frac{\left|\overrightarrow{p_{f}}\right| m_{t}^{2}}{128 \pi S^{5 / 2}}\left|\frac{\lambda_{t} \alpha_{3} c_{\chi_{1} \chi_{i} h} F\left(S / m_{t}^{2}\right)}{4 \pi}\right|^{2} g\left(S, m_{1}, m_{i}\right)
$$

where

$$
\begin{aligned}
g\left(S, m_{1}, m_{2}\right) & =\frac{\left(1-\frac{\left(m_{1}+m_{2}\right)^{2}}{S}\right)}{\left(1-\frac{m_{h}^{2}}{S}\right)^{2}} \\
F(x) & =2 \sqrt{2}\left(1+\left(1-\frac{4}{x}\right)\left[\sin ^{-1} \sqrt{\frac{x}{4}}\right]^{2}\right)
\end{aligned}
$$

and $c_{\chi_{1} \chi_{i} h}$ is the coupling between Higgs and $\chi_{1} \chi_{i}(i=2,3)$ defined in the same way as $c_{h}$ in (2.4):

$$
\begin{aligned}
\mathcal{L} & \supset c_{\chi_{1} \chi_{i} h} h \bar{\psi}_{\chi_{1}} \psi_{\chi_{i}} \\
c_{\chi_{1} \chi_{i} h} & =\frac{1}{\sqrt{2}}\left(\hat{k}_{u}\left(U_{1,1}^{*} U_{i, 2}^{*}+U_{1,2}^{*} U_{i, 1}^{*}\right)+\hat{k}_{d}\left(U_{1,1}^{*} U_{i, 3}^{*}+U_{1,3}^{*} U_{i, 1}^{*}\right)\right)
\end{aligned}
$$


Open Access. This article is distributed under the terms of the Creative Commons Attribution License (CC-BY 4.0), which permits any use, distribution and reproduction in any medium, provided the original author(s) and source are credited.

\section{References}

[1] S.P. Martin, A supersymmetry primer, Adv. Ser. Direct. High Energy Phys. 18 (1998) 1 [hep-ph/9709356] [INSPIRE].

[2] E. Halkiadakis, G. Redlinger and D. Shih, Status and implications of beyond-the-standard-model searches at the LHC, Ann. Rev. Nucl. Part. Sci. 64 (2014) 319 [arXiv: 1411.1427] [INSPIRE].

[3] P. Draper and H. Rzehak, A review of Higgs mass calculations in supersymmetric models, Phys. Rept. 619 (2016) 1 [arXiv:1601.01890] [INSPIRE].

[4] C. Cheung, L.J. Hall, D. Pinner and J.T. Ruderman, Prospects and blind spots for neutralino dark matter, JHEP 05 (2013) 100 [arXiv:1211.4873] [INSPIRE].

[5] T. Han, Z. Liu and A. Natarajan, Dark matter and Higgs bosons in the MSSM, JHEP 11 (2013) 008 [arXiv:1303.3040] [INSPIRE].

[6] A. Crivellin, M. Hoferichter, M. Procura and L.C. Tunstall, Light stops, blind spots and isospin violation in the MSSM, JHEP 07 (2015) 129 [arXiv: 1503.03478] [INSPIRE].

[7] R. Mahbubani and L. Senatore, The minimal model for dark matter and unification, Phys. Rev. D 73 (2006) 043510 [hep-ph/0510064] [INSPIRE].

[8] J. Hisano, K. Nakayama, S. Sugiyama, T. Takesako and M. Yamanaka, WIMP dark matter in gauge-mediated SUSY breaking models and its phenomenology, Phys. Lett. B 691 (2010) 46 [arXiv: 1003.3648] [INSPIRE].

[9] T. Cohen, J. Kearney, A. Pierce and D. Tucker-Smith, Singlet-doublet dark matter, Phys. Rev. D 85 (2012) 075003 [arXiv: 1109.2604] [INSPIRE].

[10] C. Cheung and D. Sanford, Simplified models of mixed dark matter, JCAP 02 (2014) 011 [arXiv:1311.5896] [INSPIRE].

[11] T. Abe, R. Kitano and R. Sato, Discrimination of dark matter models in future experiments, Phys. Rev. D 91 (2015) 095004 [arXiv:1411.1335] [InSPIRE].

[12] L. Calibbi, A. Mariotti and P. Tziveloglou, Singlet-doublet model: dark matter searches and LHC constraints, JHEP 10 (2015) 116 [arXiv:1505.03867] [INSPIRE].

[13] A. Freitas, S. Westhoff and J. Zupan, Integrating in the Higgs portal to fermion dark matter, JHEP 09 (2015) 015 [arXiv: 1506.04149] [INSPIRE].

[14] S. Banerjee, S. Matsumoto, K. Mukaida and Y.-L.S. Tsai, WIMP dark matter in a well-tempered regime: a case study on singlet-doublets fermionic WIMP, JHEP 11 (2016) 070 [arXiv: 1603.07387] [INSPIRE].

[15] T. Moroi and Y. Okada, Radiative corrections to Higgs masses in the supersymmetric model with an extra family and antifamily, Mod. Phys. Lett. A 7 (1992) 187 [INSPIRE].

[16] T. Moroi and Y. Okada, Upper bound of the lightest neutral Higgs mass in extended supersymmetric standard models, Phys. Lett. B 295 (1992) 73 [INSPIRE]. 
[17] K.S. Babu, I. Gogoladze and C. Kolda, Perturbative unification and Higgs boson mass bounds, hep-ph/0410085 [INSPIRE].

[18] K.S. Babu, I. Gogoladze, M.U. Rehman and Q. Shafi, Higgs boson mass, sparticle spectrum and little hierarchy problem in extended MSSM, Phys. Rev. D 78 (2008) 055017 [arXiv:0807.3055] [INSPIRE].

[19] S.P. Martin, Extra vector-like matter and the lightest Higgs scalar boson mass in low-energy supersymmetry, Phys. Rev. D 81 (2010) 035004 [arXiv:0910.2732] [InSPIRE].

[20] P.W. Graham, A. Ismail, S. Rajendran and P. Saraswat, A little solution to the little hierarchy problem: a vector-like generation, Phys. Rev. D 81 (2010) 055016 [arXiv: 0910.3020] [INSPIRE].

[21] S.P. Martin, Raising the Higgs mass with Yukawa couplings for isotriplets in vector-like extensions of minimal supersymmetry, Phys. Rev. D 82 (2010) 055019 [arXiv:1006.4186] [INSPIRE].

[22] S.P. Martin, Quirks in supersymmetry with gauge coupling unification, Phys. Rev. D 83 (2011) 035019 [arXiv: 1012.2072] [INSPIRE].

[23] M. Asano, T. Moroi, R. Sato and T.T. Yanagida, Non-anomalous discrete R-symmetry, extra matters and enhancement of the lightest SUSY Higgs mass, Phys. Lett. B 705 (2011) 337 [arXiv:1108.2402] [INSPIRE].

[24] M. Endo, K. Hamaguchi, S. Iwamoto and N. Yokozaki, Higgs mass and muon anomalous magnetic moment in supersymmetric models with vector-like matters, Phys. Rev. D 84 (2011) 075017 [arXiv:1108.3071] [INSPIRE].

[25] J.L. Evans, M. Ibe and T.T. Yanagida, Probing extra matter in gauge mediation through the lightest Higgs boson mass, arXiv:1108.3437 [INSPIRE].

[26] T. Moroi, R. Sato and T.T. Yanagida, Extra matters decree the relatively heavy Higgs of mass about $125 \mathrm{GeV}$ in the supersymmetric model, Phys. Lett. B 709 (2012) 218 [arXiv:1112.3142] [INSPIRE].

[27] M. Endo, K. Hamaguchi, S. Iwamoto and N. Yokozaki, Higgs mass, muon g- 2 and LHC prospects in gauge mediation models with vector-like matters, Phys. Rev. D 85 (2012) 095012 [arXiv: 1112.5653] [INSPIRE].

[28] M. Endo, K. Hamaguchi, S. Iwamoto and N. Yokozaki, Vacuum stability bound on extended GMSB models, JHEP 06 (2012) 060 [arXiv:1202.2751] [INSPIRE].

[29] S.P. Martin and J.D. Wells, Implications of gauge-mediated supersymmetry breaking with vectorlike quarks and $a \sim 125$ GeV Higgs boson, Phys. Rev. D 86 (2012) 035017 [arXiv: 1206.2956] [INSPIRE].

[30] M. Endo, K. Hamaguchi, K. Ishikawa, S. Iwamoto and N. Yokozaki, Gauge mediation models with vectorlike matters at the LHC, JHEP 01 (2013) 181 [arXiv:1212.3935] [INSPIRE].

[31] S. Iwamoto, Supersymmetry after the Higgs discovery and its LHC phenomenology, arXiv: 1305.0790 [INSPIRE].

[32] Y. Nakai, M. Reece and R. Sato, SUSY Higgs mass and collider signals with a hidden valley, JHEP 03 (2016) 143 [arXiv: 1511.00691] [INSPIRE].

[33] M. Abdullah and J.L. Feng, Reviving bino dark matter with vectorlike fourth generation particles, Phys. Rev. D 93 (2016) 015006 [arXiv:1510.06089] [INSPIRE]. 
[34] M. Abdullah, J.L. Feng, S. Iwamoto and B. Lillard, Heavy bino dark matter and collider signals in the MSSM with vectorlike fourth-generation particles, Phys. Rev. D 94 (2016) 095018 [arXiv: 1608.00283] [INSPIRE].

[35] LUX collaboration, D.S. Akerib et al., Results from a search for dark matter in the complete LUX exposure, Phys. Rev. Lett. 118 (2017) 021303 [arXiv: 1608.07648] [INSPIRE].

[36] ICECube collaboration, M.G. Aartsen et al., Improved limits on dark matter annihilation in the Sun with the 79-string IceCube detector and implications for supersymmetry, JCAP 04 (2016) 022 [arXiv: 1601.00653] [INSPIRE].

[37] XENON collaboration, E. Aprile et al., Physics reach of the XENON1T dark matter experiment, JCAP 04 (2016) 027 [arXiv: 1512.07501] [INSPIRE].

[38] LZ collaboration, D.S. Akerib et al., LUX-ZEPLIN (LZ) conceptual design report, arXiv: 1509.02910 [INSPIRE].

[39] Fermi-LAT collaboration, M. Ackermann et al., Searching for dark matter annihilation from Milky Way dwarf spheroidal galaxies with six years of Fermi Large Area Telescope data, Phys. Rev. Lett. 115 (2015) 231301 [arXiv: 1503.02641] [INSPIRE].

[40] CMS collaboration, Search for dark matter, extra dimensions and unparticles in monojet events in proton-proton collisions at $\sqrt{s}=8 \mathrm{TeV}$, Eur. Phys. J. C 75 (2015) 235 [arXiv: 1408.3583] [INSPIRE].

[41] ATLAS collaboration, Search for new phenomena in final states with an energetic jet and large missing transverse momentum in pp collisions at $\sqrt{s}=8 \mathrm{TeV}$ with the ATLAS detector, Eur. Phys. J. C 75 (2015) 299 [Erratum ibid. C 75 (2015) 408] [arXiv:1502.01518] [INSPIRE].

[42] CMS collaboration, Search for dark matter and large extra dimensions in pp collisions yielding a photon and missing transverse energy, Phys. Rev. Lett. 108 (2012) 261803 [arXiv: 1204.0821] [INSPIRE].

[43] ATLAS collaboration, Search for dark matter candidates and large extra dimensions in events with a photon and missing transverse momentum in pp collision data at $\sqrt{s}=7 \mathrm{TeV}$ with the ATLAS detector, Phys. Rev. Lett. 110 (2013) 011802 [arXiv:1209.4625] [INSPIRE].

[44] F. Staub, SARAH 4: a tool for (not only SUSY) model builders, Comput. Phys. Commun. 185 (2014) 1773 [arXiv:1309.7223] [InSPIRE].

[45] W. Porod, SPheno, a program for calculating supersymmetric spectra, SUSY particle decays and SUSY particle production at $e^{+} e^{-}$colliders, Comput. Phys. Commun. 153 (2003) 275 [hep-ph/0301101] [INSPIRE].

[46] W. Porod and F. Staub, SPheno 3.1: extensions including flavour, CP-phases and models beyond the MSSM, Comput. Phys. Commun. 183 (2012) 2458 [arXiv:1104.1573] [InSPIRE].

[47] G. Bélanger, F. Boudjema, A. Pukhov and A. Semenov, MicrOMEGAs4.1: two dark matter candidates, Comput. Phys. Commun. 192 (2015) 322 [arXiv:1407.6129] [INSPIRE].

[48] M. Cirelli, E. Del Nobile and P. Panci, Tools for model-independent bounds in direct dark matter searches, JCAP 10 (2013) 019 [arXiv:1307.5955] [INSPIRE].

[49] A. De Simone, G.F. Giudice and A. Strumia, Benchmarks for dark matter searches at the LHC, JHEP 06 (2014) 081 [arXiv: 1402.6287] [INSPIRE]. 
[50] J.F. Gunion, H.E. Haber, G.L. Kane and S. Dawson, The Higgs hunter's guide, Front. Phys. 80 (2000) 1 [INSPIRE].

[51] M.D. Goodsell, K. Nickel and F. Staub, Two-loop Higgs mass calculations in supersymmetric models beyond the MSSM with SARAH and SPheno, Eur. Phys. J. C 75 (2015) 32 [arXiv:1411.0675] [INSPIRE].

[52] M. Goodsell, K. Nickel and F. Staub, Generic two-loop Higgs mass calculation from a diagrammatic approach, Eur. Phys. J. C 75 (2015) 290 [arXiv:1503.03098] [INSPIRE].

[53] L.J. Hall, D. Pinner and J.T. Ruderman, A natural SUSY Higgs near $126 \mathrm{GeV}$, JHEP 04 (2012) 131 [arXiv:1112.2703] [InSPIRE].

[54] R. Kitano and Y. Nomura, A solution to the supersymmetric fine-tuning problem within the MSSM, Phys. Lett. B 631 (2005) 58 [hep-ph/0509039] [INSPIRE].

[55] G. Jungman, M. Kamionkowski and K. Griest, Supersymmetric dark matter, Phys. Rept. 267 (1996) 195 [hep-ph/9506380] [INSPIRE].

[56] Planck collaboration, P.A.R. Ade et al., Planck 2015 results. XIII. Cosmological parameters, Astron. Astrophys. 594 (2016) A13 [arXiv: 1502.01589] [INSPIRE].

[57] N. Zhou, D. Berge and D. Whiteson, Mono-everything: combined limits on dark matter production at colliders from multiple final states, Phys. Rev. D 87 (2013) 095013 [arXiv: 1302.3619] [INSPIRE].

[58] O. Buchmueller, M.J. Dolan, S.A. Malik and C. McCabe, Characterising dark matter searches at colliders and direct detection experiments: vector mediators, JHEP 01 (2015) 037 [arXiv: 1407.8257] [INSPIRE].

[59] J. Alwall et al., The automated computation of tree-level and next-to-leading order differential cross sections and their matching to parton shower simulations, JHEP 07 (2014) 079 [arXiv: 1405.0301] [INSPIRE].

[60] R.D. Ball et al., Parton distributions with LHC data, Nucl. Phys. B 867 (2013) 244 [arXiv: 1207.1303] [INSPIRE].

[61] U. Ellwanger, C. Hugonie and A.M. Teixeira, The next-to-minimal supersymmetric standard model, Phys. Rept. 496 (2010) 1 [arXiv:0910.1785] [INSPIRE].

[62] P. Batra, A. Delgado, D.E. Kaplan and T.M.P. Tait, The Higgs mass bound in gauge extensions of the minimal supersymmetric standard model, JHEP 02 (2004) 043 [hep-ph/0309149] [INSPIRE].

[63] A. Maloney, A. Pierce and J.G. Wacker, D-terms, unification and the Higgs mass, JHEP 06 (2006) 034 [hep-ph/0409127] [INSPIRE].

[64] G. Bélanger, F. Boudjema, A. Pukhov and A. Semenov, MicrOMEGAs_3: a program for calculating dark matter observables, Comput. Phys. Commun. 185 (2014) 960 [arXiv: 1305.0237] [INSPIRE]. 